\title{
Person-centred care in osteoarthritis and inflammatory arthritis: a scoping review of people's needs outside of healthcare
}

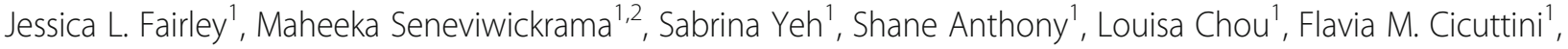
Kaye Sullivan ${ }^{3}$, Andrew M. Briggs ${ }^{4}$ and Anita E. Wluka ${ }^{1 *}$ (id

\begin{abstract}
Background: Arthritis, regardless of cause, has significant physical, social and psychological impacts on patients. We aimed to identify the non-healthcare needs perceived by patients with inflammatory arthritis (IA) and osteoarthritis $(\mathrm{OA})$, and to determine if these differ.

Methods: We electronically searched MEDLINE, PsycINFO, EMBASE and CINAHL (1990-2020) systematically to identify non-healthcare-related needs of people with IA or OA. All citations were screened and quality appraised by two reviewers. Data was extracted by a single reviewer.

Results: The search identified 7853 citations, with 31 studies included (12 for OA, 20 for IA). Six areas of need emerged and these were similar in both group These were: 1) Assistance with activities of daily living especially related to a lack of independence; 2) Social connectedness: need for social participation; 3) Financial security: worry about financial security and increased costs of health-seeking behaviours; 4) Occupational needs: desire to continue work for financial and social reasons, facilitated by flexibility of workplace conditions/environment; 5) Exercise and leisure: including limitation due to pain; 6) Transportation: limitations in ability to drive and take public transport due to mobility concerns. Many areas of need were linked; e.g. loss of employment and requiring support from family was associated with a sense of "failure" and loss of identity, as social isolation.

Conclusions: This review highlights the pervasive impact of arthritis on peoples' lives, regardless of aetiology, albeit with a limited evidence base. Improved identification and targeting of non-healthcare needs of people with arthritis is likely to improve person-centred care.
\end{abstract}

Keywords: Arthritis, Osteoarthritis, Person centred care, Rheumatoid arthritis, Patient needs

\section{Significance and innovations}

- People with osteoarthritis and inflammatory arthritis perceive needs for support in many areas of life outside of direct healthcare, related to activities of

\footnotetext{
* Correspondence: anita.wluka@monash.edu

${ }^{1}$ Department of Epidemiology and Preventative Medicine, School of Public Health and Preventative Medicine, Monash University, Melbourne, Victoria, Australia

Full list of author information is available at the end of the article
}

daily living, exercise, social participation, environment, occupation and transport.

- Despite differences in pathology, the nonhealthcare-related needs of people with OA and IA are similar.

- Improved identification and targeting of nonhealthcare needs of people with arthritis is likely to improve person-centred care.

C The Author(s). 2021 Open Access This article is licensed under a Creative Commons Attribution 4.0 International License, which permits use, sharing, adaptation, distribution and reproduction in any medium or format, as long as you give appropriate credit to the original author(s) and the source, provide a link to the Creative Commons licence, and indicate if changes were made. The images or other third party material in this article are included in the article's Creative Commons licence, unless indicated otherwise in a credit line to the material. If material is not included in the article's Creative Commons licence and your intended use is not permitted by statutory regulation or exceeds the permitted use, you will need to obtain permission directly from the copyright holder. To view a copy of this licence, visit http://creativecommons.org/licenses/by/4.0/. The Creative Commons Public Domain Dedication waiver (http://creativecommons.org/publicdomain/zero/1.0/) applies to the data made available in this article, unless otherwise stated in a credit line to the data. 


\section{Background}

Arthritis affects up to $80 \%$ of older adults in developed countries [1], with the two most common forms being osteoarthritis (OA) and rheumatoid arthritis (RA). Osteoarthritis accounts for $2.19 \%$ of all years lived with disability (YLDs) for any condition worldwide; while RA accounts for $0.28 \%$ of all YLDs [2]. The management of OA is limited with no disease modifying therapy available; the focus of therapy is symptom control and maintenance of function, with joint replacement an option for end-stage disease. For people living with OA, creating a healthcare and broader social environment that supports symptom management and capabilities to effectively self-manage a long-term disease is essential [3]. In contrast, biological therapies have improved morbidity and mortality outcomes in people with inflammatory arthritis (IA), particularly (RA) [4]. With effective treatments available for controlling disease activity, medical practitioners may focus care priorities in this area and other specific health needs (e.g. co-morbidity care), rather than broader health and social needs that may be relevant to the person. Notably, in people with other rheumatologic conditions, including lower back pain, addressing needs outside of direct medical care to provide holistic care, can improve quality of life [5].

While disease management may necessarily vary between individuals, all form of arthritis are associated with common impacts on people's lives, in particular a loss of function, loss of dexterity, mental health sequelae and limitations in participation, often leading to unfavourable social consequences [6-8]. While these domains of impact are common, the nature of the impacts will vary between by disease. For example, those with inflammatory arthritis taking immunosuppressant medication may have specific occupational- and transportrelated needs, to avoid situations that increase their risk of infection which are not relevant to those with osteoarthritis. To optimise holistic care, it is necessary to understand peoples' non-healthcare related needs, beyond direct healthcare provision, such as the social determinants of health [9]. Accordingly, we aimed to identify current knowledge regarding the non-healthcare needs perceived by patients with OA and IA, and examine differences where identified.

\section{Methods}

A systematic scoping review was performed to provide an overview of the literature around the patient perceived non-healthcare needs of people with OA and IA, in line with the PRISMA guidelines for scoping reviews [10]. This was conducted within a larger project examining patients' perceived needs relating to musculoskeletal health [11].

\section{Search strategy}

Medline, EMBASE, CINAHL and PsycINFO were electronically searched (1990 to September 2020) using a combination of keywords and $\mathrm{MeSH}$ terms related to perceived non-healthcare needs in people with $\mathrm{OA}$ and IA separately. A comprehensive search strategy was codeveloped iteratively by a multidisciplinary team involving an academic librarian, input from a patient representative and four clinician researchers (Rheumatologists, Physiotherapist and public health physician) (Supplement Material Figures S1a, S1b, S2a, S2b, S3a, S3b, S4a, S4b).

\section{Inclusion and exclusion criteria}

English-language studies were included examining people older than 18 years with OA and IA. IA was defined as any joint disease where the primary mechanism was inflammation or synovitis, including RA, systemic lupus erythematosus (SLE) and psoriatic arthritis (PsA), excluding OA or crystal arthritis. Regarding OA, both clinical and radiological definitions were included. Studies had to report on perceived non-healthcare needs relevant to OA or IA. The concept of a "need" is complex, and currently without a consensus definition in the literature [12]. Broadly, we defined "need" in the context of health as a person's desires, expectations and requirements [12], aligned with other work in this space [11, 13]. Non-healthcare services were defined as interventions, supports or structures not directly relating to healthcare, to support and assist people with functional limitation from their disease. Full text articles were included; no restrictions on study design were made to ensure a broad focus was maintained.

\section{Study selection}

Each title and abstract was screened for eligibility independently by 2 investigators (MS, SY, LC or JF). Full texts of studies meeting the inclusion criteria were retrieved and assessed (MS, LC or JF). Discrepancies were reviewed with an additional investigator (AW) to reach consensus.

\section{Data extraction and analysis}

Data were extracted by one author (MS, LC or JF) using a standardised data extraction form designed to capture demographic data, aims and description of study methods and outcomes. Included studies were reviewed by one author (MS, JF or AW) to identify aspects of non-healthcare needs, using principles of metaethnography to synthesise qualitative data [14]; the most common analytic approach for synthesising qualitative data from primary studies [15]. The principle of metaethnography is to empirically derive, though an inductive analytic frame, new concepts, interpretations, or theories 
that extend or go beyond findings of any individual study. In this way, a body of qualitative evidence contributes to the development of new themes or concepts [16]. The analytic approach used in this review followed the seven phases described by Noblit and Hare [17] and more recently described by France et al. [18]. Initially, one author (MS) reviewed each study in detail, reading the text several times to inductively derive an overarching framework of concepts and underlying themes from the yield of the qualitative studies. This framework was directly informed by data extracted from the primary studies and any pertinent points raised by the authors of the primary studies in the discussion, as recommended by France et al. [18]. In developing the framework of themes and subthemes each primary study was compared to the others to identify comparability, similarity or opposition based on grouping concepts. Once primary studies were translated into each other two senior rheumatologists (FC, AW) independently reviewed the framework of concepts and themes to ensure clinical meaningfulness and face validity.
Quantitative meta-analysis was not possible due to nature and heterogeneity of included studies. For this reason, quantitative data were reported narratively.

\section{Risk of Bias assessments}

Risk of bias assessments were performed independently by 2 investigators (JF, SA, AW or SY). Discrepancies were reviewed by a third investigator (AW) to reach consensus (Supplementary Material Table S1, S2). For qualitative studies, the Critical Appraisal Skills Programme (CASP) tool was used to assign risk of bias estimates [19]. For quantitative articles, the method described by Hoy et al. (Supplementary Material Appendix S1) was used to assess internal and external validity of studies [20]. Studies were deemed to be at low risk of bias if scoring eight or more "yes" answers, at moderate risk if scoring six to seven, and at high risk of bias when scoring less than six [20]. For qualitative studies, the Critical Appraisal Skills Programme (CASP) tool was used to assign risk of bias estimates [10].

7853 studies identified by search strategy

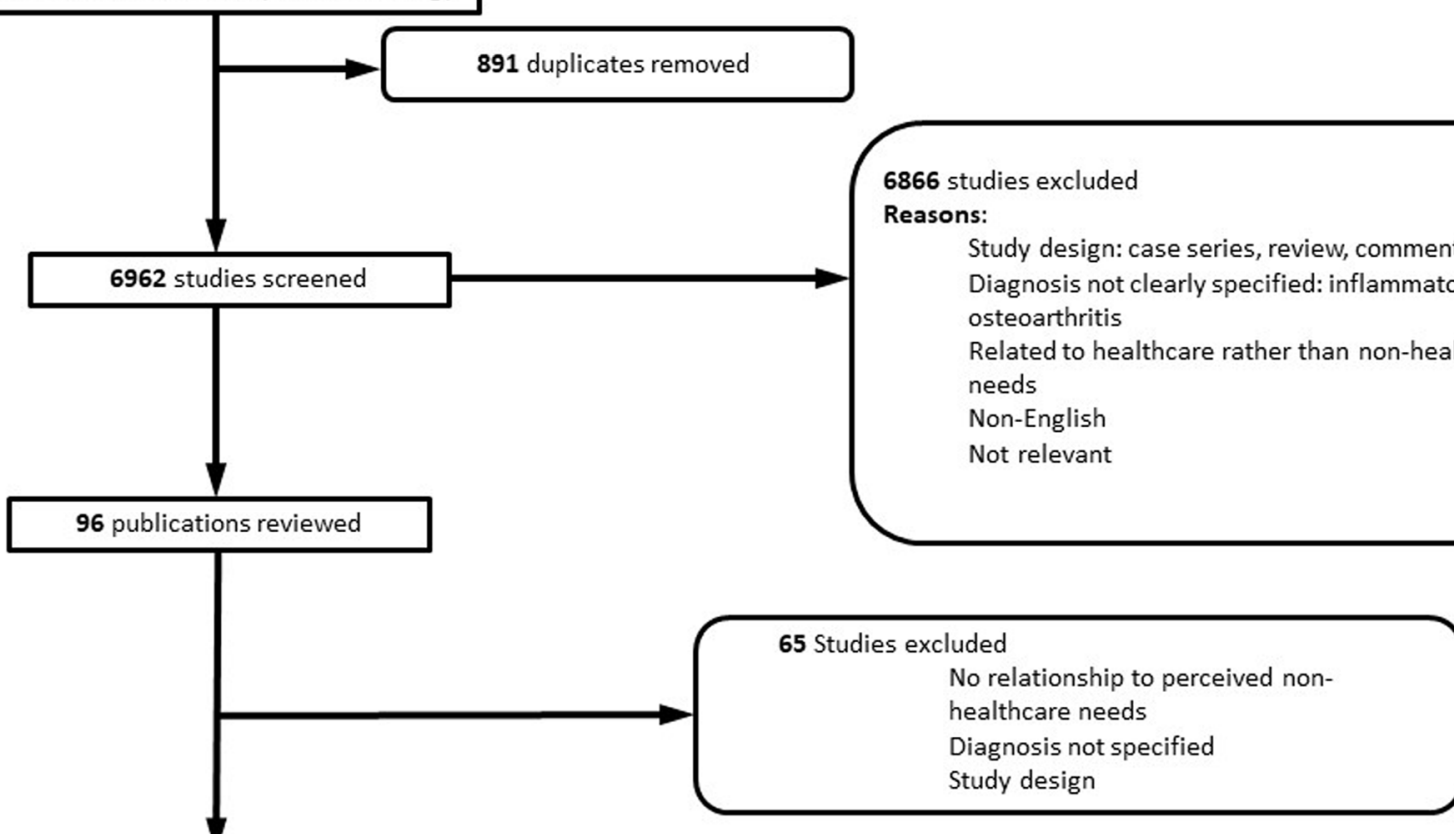

$\mathbf{3 1}$ studies included

OA: 12 studies

IA: 20 studies

(1 study for both)

Fig. 1 PRISMA Diagram 


\section{Results}

The search strategy identified 7853 potentially relevant abstracts, of which 891 were excluded as duplicates, and 6962 excluded after abstract screening (Fig. 1: PRISMA Diagram). Ninety-six manuscripts were retrieved for review, with 31 included in the final review. Twelve (39\%) studies related to OA, and $20(65 \%)$ related to IA, with one study involving participants with OA and IA with outcomes reported separately. Description of the included studies is presented in Table 1.

Twelve studies investigated the perceived nonhealthcare needs of people with OA [21-32]. Included studies were all from high income countries except one (Nigeria [26]), involving 17-362 participants. Mean age ranged from 49.6 to 72.4 years, with studies including predominantly women (60-100\% female). Participants were recruited from the ambulatory care settings in all studies; usually outpatient clinics (either rheumatology or orthopaedic [22, 28, 30,31] or primary care [25].

Five studies included people with knee OA $[22,25,26$, $28,32]$, four hand OA [24, 27, 29, 30], two a mixture of joint involvement $[21,23]$ and one did not specify [31]. OA was usually diagnosed clinically $[21,22,24-27,29-$ $32]$. OA was mild in two studies [28, 30] and end-stage in another [22] but most commonly severity was unspecified $(9 / 12,75 \%)$. Six studies used qualitative methods (semi-structured interviews [24, 25, 27, 28] or focus group discussions [22, 23], five quantitative methods [21, 26, 30-32], and one both [29]. Of qualitative studies, five were assessed as low risk of bias [22, 24, $25,27,28$ ] and one moderate risk of bias [23] (Supplementary Material Table S1). Of quantitative studies, five were assessed as moderate risk of bias [21, 26, 29, 30, 32], and one at high risk of bias [31] (Supplementary Material Table S2).

Twenty studies discussed non-healthcare needs for IA [31, 33-51]. All but one study included predominantly female participants $(58-100 \%)$ [41], with all studies from high-income countries. Included participants were generally over 50 years of age [31, 33-40, 42-45, 48, 49, 51], although in four studies mean age was in the 40s [41, $46,47,50]$. IA was usually diagnosed clinically [31, 33, $35-37,39,40,42-50]$. RA was the most common diagnosis [34, 35, 37-40, 42-48, 50, 51] [33, 49], with participants usually recruited from outpatient clinics [31, 35, $36,38,40,41,43-45,48-50]$. Nine quantitative studies used questionnaires [31, 33, 37, 38, 40, 44, 46, 47, 51], while 11 qualitative studies used interviews [34-36, 39, $41,42,45,48-50]$ or focus groups [43]. Of the qualitative studies, six were assessed as low risk of bias [34, 42, $45,48-50]$ and five at moderate risk of bias [35, 36, 39, 41, 43] (Supplementary Material Table S1). Of the quantitative studies, one was assessed as low risk of bias [38], two at moderate risk of bias $[44,46]$ and six at high risk of bias [31, 33, 37, 40, 47, 51] (Supplementary Material Table S2).

\section{Areas of need identified}

This review identified six key areas of need in people with IA or OA, common to both groups (Table 2, Table 3).

1) Activities of daily living (ADLs) both inside and outside the home

Ten OA studies (Table 2) [21, 22, 24-29, 31, 32] and 15 IA studies (Table 3) [23, 31, 33-37, 39, 42-48] discussed difficulties facing people regarding ADLs.

ADLs of people with OA were limited by symptoms [21, 32], including pain [28]. People with OA worried about needing help from others [21, 22, 24, 25, 28, 31]: $75 \%$ in one study [31]. For household chores, help was required either from within the family or external sources [22, 24, 25, 27, 28]. This sometimes contributed to a sense of "failure" to fulfil social obligations e.g. caring for children or partners [22]. People acknowledged that they may struggle to live alone [24]. Cleanliness and grooming were important to people with hand OA [24], who experienced difficulty with fine motor tasks including buttons and lacing shoes [27]. Moreover, hand OA limited the dexterity required for cell phones, cutlery and some payment systems (e.g. coins and notes) [24, 27]. Gadgets with an accessible design and assistive devices improved daily functioning $[24,29]$.

People with IA frequently received assistance with ADLs [31, 34, 35, 39, 42-45, 48]; up to $93 \%$ of people with RA [31]. Partners [42] or children [45] most commonly provided help. Participants disliked accepting assistance from children [34]. Daily activities, particularly housework, were difficult with IA [33, 34, 36, 37, $46,51]$; IA was associated with less enjoyment of life [37]. Pain and stiffness contributed to functional limitations [36, 48]. Functional difficulties could make people feel anxious, frustrated or "like a failure" [33]. Participants often lived alone (29\%) [44] and worried about inability to obtain assistance when required [31]. Participants wanted to do more than they could [35]. Compared to amputees, people with IA were less independent and well-adjusted to their circumstances [23]. Participants wanted to tools to make ADLs easier [39], especially environmental modifications. Areas in the home, workplace and outdoors required modifications [23, 40]. Participants valued security and worried about falling victim to crime due to perceived invalidity [44].

2) Social participation needs 
Table 1 Included manuscripts relating to scoping review of consumer perceived other service needs related to osteoarthritis and inflammatory arthritis, including rheumatoid arthritis and other inflammatory conditions

\begin{tabular}{|c|c|c|c|c|c|c|}
\hline \multicolumn{7}{|l|}{ Osteoarthritis } \\
\hline $\begin{array}{l}\text { Author (year) } \\
\text { Country }\end{array}$ & Diagnosis & $\begin{array}{l}\text { No. of } \\
\text { subjects }\end{array}$ & Source of participants & Age \& Gender & Aim & $\begin{array}{l}\text { Study type/ } \\
\text { design }\end{array}$ \\
\hline $\begin{array}{l}\text { Ackerman } \\
(2013)[21] \\
\text { Australia } \\
\left.\text { (High }_{\text {income }}{ }^{a}\right)\end{array}$ & $\begin{array}{l}\text { ACR criteria or } \\
\text { radiology } \\
\text { reports }\end{array}$ & $\begin{array}{l}N=126 \\
\text { Hip OA: } 31 \% \\
\text { Knee OA: } 63 \% \\
\text { Hip + Knee } \\
\text { OA: } 6 \%\end{array}$ & $\begin{array}{l}\text { Rheumatology or Orthopaedic } \\
\text { outpatient clinic (mixed public } \\
\text { and private hospitals) }\end{array}$ & $\begin{array}{l}\text { Age(median): } 67 \\
\text { years (IQR 57-73) } \\
\text { Female } 60 \%\end{array}$ & $\begin{array}{l}\text { To understand barriers to } \\
\text { participation in community- } \\
\text { based arthritis self- } \\
\text { management programs and } \\
\text { patient preferences for self- } \\
\text { management education. }\end{array}$ & $\begin{array}{l}\text { Quantitative } \\
\text { Questionnaire }\end{array}$ \\
\hline $\begin{array}{l}\text { Al-Taiar } \\
\text { (2013) [22] } \\
\text { Kuwait (High } \\
\text { income }^{\text {a }} \text { ) }\end{array}$ & Clinical & $\begin{array}{l}N=39 \\
\text { Knee OA }\end{array}$ & $\begin{array}{l}\text { Waiting list for TKIR for severe } \\
\text { knee OA in the only public } \\
\text { orthopaedic hospital in Kuwait }\end{array}$ & $\begin{array}{l}\text { Age (mean): } 62.5 \\
+/-7.9 \text { years } \\
\text { Female } 100 \%\end{array}$ & $\begin{array}{l}\text { To explore the pain } \\
\text { experience and mobility } \\
\text { limitations as well as the } \\
\text { patient's decision making } \\
\text { process to undertake knee } \\
\text { joint replacement }\end{array}$ & $\begin{array}{l}\text { Qualitative, } \\
\text { focus group } \\
\text { discussions }\end{array}$ \\
\hline $\begin{array}{l}\text { Baumann } \\
\text { (2007) [23] } \\
\text { France (High } \\
\left.\text { income }^{\text {a }}\right)\end{array}$ & $\begin{array}{l}\text { Diagnosis } \\
\text { methods not } \\
\text { specified }\end{array}$ & $\begin{array}{l}N=96 \\
\text { Knee OA 66\% } \\
\text { Finger OA } \\
50 \% \\
\text { Hip OA 46\% }\end{array}$ & $\begin{array}{l}\text { Customers of } 10 \text { pharmacies } \\
\text { in } 10 \text { towns in } 10 \text { regions } \\
\text { randomly selected from } 22 \\
\text { French regions. The first } 10 \\
\text { customers who came to } \\
\text { purchase any medication. }\end{array}$ & $\begin{array}{l}\text { Age(mean): } 65 \\
\text { years (range } 42- \\
89 \text { years) } \\
\text { Female } 81 \%\end{array}$ & $\begin{array}{l}\text { To understand the } \\
\text { expectations of patients with } \\
\text { OA to use these to improve } \\
\text { healthcare provision and the } \\
\text { doctor-patient relationship }\end{array}$ & $\begin{array}{l}\text { Qualitative } \\
\text { Focus groups }\end{array}$ \\
\hline $\begin{array}{l}\text { Bukhave \& } \\
\text { Huniche } \\
(2014)[24] \\
\text { Denmark } \\
(\text { High } \\
\left.\text { income }^{\mathrm{a}}\right)\end{array}$ & Clinical & $\begin{array}{l}N=31 \\
\text { Hand OA }\end{array}$ & $\begin{array}{l}\text { Referred by a doctor or } \\
\text { volunteers (via an article in } \\
\text { Danish Rheumatism } \\
\text { Association magazine) }\end{array}$ & $\begin{array}{l}\text { Age(mean): } 62.9 \\
\text { years (range } 38- \\
89 \text { years) } \\
\text { Female } 84 \%\end{array}$ & $\begin{array}{l}\text { To explore perspectives on } \\
\text { activities and participation in } \\
\text { everyday life among people } \\
\text { with hand OA }\end{array}$ & $\begin{array}{l}\text { Qualitative, } \\
\text { semi-structured } \\
\text { interviews }\end{array}$ \\
\hline $\begin{array}{l}\text { Chan (2011) } \\
{[25]} \\
\text { Hong Kong } \\
(\text { High } \\
\left.\text { income }^{\mathrm{a}}\right)\end{array}$ & $\begin{array}{l}\text { Clinical (ACR } \\
\text { Criteria) }\end{array}$ & $\begin{array}{l}N=20 \\
\text { Knee OA }\end{array}$ & GP clinic & $\begin{array}{l}\text { Age(mean): } 57.05 \\
+/- \text { SD } 10.79 \\
\text { years } \\
\text { Female } 65 \%\end{array}$ & $\begin{array}{l}\text { To evaluate the influence of } \\
\text { pain patterns on quality of } \\
\text { life, and to investigate } \\
\text { interpretation and coping } \\
\text { strategies }\end{array}$ & $\begin{array}{l}\text { Qualitative, } \\
\text { semi-structured } \\
\text { interviews }\end{array}$ \\
\hline $\begin{array}{l}\text { llori (2016) } \\
{[26]} \\
\text { Nigeria } \\
\left(\text { Lower-Middle }^{\text {Income }}{ }^{\mathrm{a}}\right)\end{array}$ & $\begin{array}{l}\text { Clinical (ACR } \\
\text { Criteria) }\end{array}$ & $\begin{array}{l}N=270 \\
\text { Knee OA }\end{array}$ & GP clinic & $\begin{array}{l}\text { Age: NR } \\
\text { Gender: NR }\end{array}$ & $\begin{array}{l}\text { To assess family and social } \\
\text { supports, and health impact } \\
\text { on patients with knee OA }\end{array}$ & $\begin{array}{l}\text { Quantitative } \\
\text { Questionnaires }\end{array}$ \\
\hline $\begin{array}{l}\text { Hill (2010) [27] } \\
\text { United } \\
\text { Kingdom } \\
\text { (High }_{\text {income }}{ }^{\mathrm{a}} \text { ) }\end{array}$ & $\begin{array}{l}\text { Clinical (by GP } \\
\text { or } \\
\text { rheumatologist) }\end{array}$ & $\begin{array}{l}N=29 \\
\text { Hand OA }\end{array}$ & $\begin{array}{l}\text { GP or rheumatology } \\
\text { outpatient clinic }\end{array}$ & $\begin{array}{l}\text { Age(mean): } \\
\text { primary } 62.4 \\
\text { years, secondary } \\
63.6 \text { years } \\
\text { Female: primary } \\
80 \% \text {, secondary } \\
93 \%\end{array}$ & $\begin{array}{l}\text { To investigate the functional } \\
\text { impact of hand OA on } \\
\text { everyday life }\end{array}$ & $\begin{array}{l}\text { Qualitative: } \\
\text { Semi- } \\
\text { structured } \\
\text { interviews }\end{array}$ \\
\hline $\begin{array}{l}\text { Kao (2014) } \\
{[28]} \\
\text { Taiwan (High } \\
\text { income }^{\text {a }} \text { ) }\end{array}$ & $\begin{array}{l}\text { Stage } 1 \text { or } 2 \\
\text { knee OA } \\
\text { (Ahlback) }\end{array}$ & $\begin{array}{l}N=17 \\
\text { Knee OA }\end{array}$ & $\begin{array}{l}\text { Orthopaedic outpatient clinic } \\
\text { ( } 2 \text { hospitals) }\end{array}$ & $\begin{array}{l}\text { Age (mean): } 49.6 \\
+ \text { +/-SD } 4.2 \text { years } \\
\text { (range } 43-55 \\
\text { years) } \\
\text { Female } 82 \%\end{array}$ & $\begin{array}{l}\text { To understand the illness } \\
\text { experiences of middle-aged } \\
\text { adults with early knee OA }\end{array}$ & $\begin{array}{l}\text { Qualitative, } \\
\text { semi-structured } \\
\text { interviews }\end{array}$ \\
\hline $\begin{array}{l}\text { Kjeken (2013) } \\
{[29]} \\
\text { Norway (High } \\
\left.\text { income }^{\mathrm{a}}\right)\end{array}$ & $\begin{array}{l}\text { Clinical (ACR } \\
\text { Criteria) }\end{array}$ & $\begin{array}{l}N=125 \\
\text { Hand OA }\end{array}$ & $\begin{array}{l}\text { Rheumatology and } \\
\text { orthopaedic outpatient clinics } \\
\text { (public hospital) }\end{array}$ & $\begin{array}{l}\text { Age(mean): } 64.5 \\
\text { years } \\
\text { Female } 98 \%\end{array}$ & $\begin{array}{l}\text { To explore self-management } \\
\text { strategies in hand OA, espe- } \\
\text { cially strategies for daily } \\
\text { activities }\end{array}$ & $\begin{array}{l}\text { Qualitative and } \\
\text { quantitative } \\
\text { Questionnaires }\end{array}$ \\
\hline $\begin{array}{l}\text { Leung et al. } \\
\text { (2019) } \\
\text { Singapore } \\
\left(\text { High }^{\mathrm{a}}\right)[30]\end{array}$ & $\begin{array}{l}\text { Clinical (ACR } \\
\text { Criteria) }\end{array}$ & $\begin{array}{l}N=45 \\
\text { Hand OA }\end{array}$ & $\begin{array}{l}\text { Rheumatology outpatient } \\
\text { clinic (dedicated hand OA } \\
\text { clinic) }\end{array}$ & $\begin{array}{l}\text { Age (mean): } \\
\text { 64.3 years (range } \\
51-82 \text { years) } \\
\text { Female } 91.1 \%\end{array}$ & $\begin{array}{l}\text { To explore patients' } \\
\text { perspectives in priorities for } \\
\text { core domains for clinical } \\
\text { trials related to hand OA. }\end{array}$ & $\begin{array}{l}\text { Quantitative } \\
\text { Questionnaires }\end{array}$ \\
\hline $\begin{array}{l}\text { Neville (1999) } \\
\text { [31] } \\
\text { Canada (High } \\
\text { income }^{a} \text { ) }\end{array}$ & Clinical & $\begin{array}{l}N=197 \\
\text { - RA: } 57 \\
\text { - SLE: } 27 \\
\text { - OA: } 41\end{array}$ & $\begin{array}{l}\text { Rheumatology outpatient } \\
\text { clinic (public or private, } \\
\text { multicentre) }\end{array}$ & $\begin{array}{l}\text { Age(mean): } 60 \\
+/-15 \text { years } \\
\text { Female } 83.2 \%\end{array}$ & $\begin{array}{l}\text { To identify concerns \& } \\
\text { learning interests of arthritis } \\
\text { patients }\end{array}$ & $\begin{array}{l}\text { Quantitative } \\
\text { Descriptive } \\
\text { cross-sectional } \\
\text { self- }\end{array}$ \\
\hline
\end{tabular}


Table 1 Included manuscripts relating to scoping review of consumer perceived other service needs related to osteoarthritis and inflammatory arthritis, including rheumatoid arthritis and other inflammatory conditions (Continued)

\begin{tabular}{|c|c|c|c|c|c|c|}
\hline & & $\begin{array}{l}\text { - Back Pain: } 55 \\
\text { - Systemic } \\
\text { sclerosis: } 17\end{array}$ & & & & $\begin{array}{l}\text { administered } \\
\text { questionnaires }\end{array}$ \\
\hline $\begin{array}{l}\text { Tanimura } \\
\text { (2011) [32] } \\
\text { Japan (high } \\
\text { income }^{\mathrm{a}} \text { ) }\end{array}$ & Clinical & $\begin{array}{l}N=362 \\
\text { Knee OA }\end{array}$ & $\begin{array}{l}\text { Orthopaedic outpatient clinics } \\
\text { (predominantly public } \\
\text { hospitals) }\end{array}$ & $\begin{array}{l}\text { Age (mean): } 72.4 \\
+/-9.6 \text { years } \\
\text { Female } 281 / 362 \\
(78 \%)\end{array}$ & $\begin{array}{l}\text { To develop an instrument to } \\
\text { assess difficulties in daily life } \\
\text { of patients with knee OA, } \\
\text { and to investigate factors } \\
\text { influencing difficulties in life }\end{array}$ & $\begin{array}{l}\text { Quantitative } \\
\text { Questionnaires }\end{array}$ \\
\hline
\end{tabular}

\section{Inflammatory arthritis}

\section{Author (year) Diagnosis Country}

Alten (2019) Clinical
[33]
Europe/
Canada (High
income $^{\text {a }}$ )

\begin{tabular}{|c|c|}
\hline $\begin{array}{l}\text { Been-Dahmen } \\
\text { (2017) [34] } \\
\text { The } \\
\text { Netherlands } \\
\text { (High }^{\text {income }}{ }^{a} \text { ) }\end{array}$ & Not specified \\
\hline
\end{tabular}

$\begin{array}{lll}\text { Bergsten } & \text { Clinical } & N=16 \\ \text { (2011) [35] } & & \text { RA } \\ \text { Sweden } & & \\ \text { (High }^{\text {income }} & & \end{array}$

Carter (2019)
[36]
Australia/
New Zealand
(High
income ${ }^{\text {a) }}$
Cunha-

Cunha-

Miranda

(2010) [37]

Portugal(High

income ${ }^{\mathrm{a}}$ )

\begin{tabular}{|c|c|c|c|}
\hline $\begin{array}{l}\text { Giacomelli } \\
(2015) \text { [38] } \\
\text { Italy }(\text { High } \\
\left.\text { income }{ }^{\mathrm{a}}\right)\end{array}$ & Not specified & $\begin{array}{l}N=743 \\
\text { - RA:327 } \\
\text { - PSA:214 } \\
\text { - AS:200 }\end{array}$ & $\begin{array}{l}\text { Rheumatology outpatient } \\
\text { department }\end{array}$ \\
\hline $\begin{array}{l}\text { Hamnes } \\
(2011)[39] \\
\text { Norway (High } \\
\text { income }^{a} \text { ) }\end{array}$ & $\begin{array}{l}\text { Clinical (GP or } \\
\text { specialist) } \\
\text { ACR criteria }\end{array}$ & $\begin{array}{l}N=16 \\
\text { - RA: } 8 \\
\text { - Fibromyalgia } \\
\text { (FM): } 8\end{array}$ & $\begin{array}{l}\text { Patients awaiting self- } \\
\text { management programmes } \\
\text { (SMP) }\end{array}$ \\
\hline $\begin{array}{l}\text { Henchoz } \\
(2013)[40] \\
\text { Switzerland } \\
\text { (High } \\
\text { income }^{\mathrm{a}} \text { ) }\end{array}$ & $\begin{array}{l}\text { Clinical } \\
\text { (Rheumatologist) } \\
\text { ACR functional } \\
\text { classes I-III }\end{array}$ & $\begin{array}{l}N=89 \\
\text { All RA }\end{array}$ & $\begin{array}{l}\text { Rheumatology outpatient } \\
\text { clinic (tertiary centre) }\end{array}$ \\
\hline $\begin{array}{l}\text { Herrera-Saray } \\
\text { (2013) [41] }\end{array}$ & $\begin{array}{l}\text { Disabled users } \\
\text { of assistive }\end{array}$ & $\begin{array}{l}N=15 \\
\text { Inflammatory }\end{array}$ & $\begin{array}{l}\text { Rheumatologist \& snow-bal } \\
\text { method }\end{array}$ \\
\hline
\end{tabular}

Rheumatology hospital; outpatient clinic or rehabilitation service

\section{No. of \\ $N=1231$ \\ RA \\ Source of participants \\ Online advertising, previous survey participants} Rheumatology outpatient
department

Age \& Gender

\section{Primary study aim}

Study type/ design

Age: most common 40-59 years (48\%), 30\% $>60$ years, $22 \%$

$<40$ years

Female 58\%

Age: most common 55-64 years $(10 / 20$;

$50 \%), 5 / 20<55$ and 5/20 65+

years.

Female 14/20

(70\%)

Age (mean): Women 62 years (Range 28-82 years), Men 61 years (range 4270 years) Female 10/16 (62.5\%)

Age (mean): 53 +/- 13 years

Female 62\%

PSA with foot

Rheumatology outpatient department

Rheumatology outpatient department

$N=233$

- RA

Age (mean)

$55.13+/-14.49$

years

Female $82.5 \%$

To determine principle sources of disease information in RA patients, unmet needs and patient involvement in decision making.

Age: 493 patients $>45$ years of age Female 58\%

Age (mean):

51.4 years

Female 13/16

(81.2\%)

Age (mean):

58.4 years Women 71/89

(79.8\%)

To patient involvement in medical decisions, quality of life and unmet needs after introducing biological therapies

To identify expectations prior to a one-week selfmanagement program, and outcomes

Qualitative: semi-structured interviews

Quantitative: Questionnaires

To examine patients' perceptions of exercise benefits, barriers, and their preferences for exercise

Age (mean): 41 years
To identify usage/ accessibility problems faced
Qualitative: interviews 14/ 20

Qualitative Face-to-face interviews
Quantitative: Questionnaires

Qualitative: Semistructured interviews

Quantitative: cross sectional study, using selfadministered questionnaire

Qualitative: Indepth 
Table 1 Included manuscripts relating to scoping review of consumer perceived other service needs related to osteoarthritis and inflammatory arthritis, including rheumatoid arthritis and other inflammatory conditions (Continued)

\begin{tabular}{|c|c|c|c|c|c|c|}
\hline $\begin{array}{l}\text { Mexico \& } \\
\text { Colombia } \\
\text { (Both Upper- } \\
\text { Middle- } \\
\text { Income }\end{array}$ & $\begin{array}{l}\text { devices } \\
\text { Not mentioned }\end{array}$ & $\begin{array}{l}\text { Arthritis 9/15 } \\
\text { - RA: } 4 \\
\text { - Spondylo- } \\
\text { arthropathy: } \\
5 \\
\text { - Amputee } \\
\text { (any cause): } 6\end{array}$ & & $\begin{array}{l}\text { Women } 6 / 15 \\
(40 \%)\end{array}$ & $\begin{array}{l}\text { by the disabled and users of } \\
\text { assistive devices, and } \\
\text { physical barriers that limit } \\
\text { their mobility }\end{array}$ & interviews \\
\hline $\begin{array}{l}\text { Kostova } \\
\text { (2014) [42] } \\
\text { Switzerland } \\
\left.\text { (High }^{\text {income }}{ }^{a}\right)\end{array}$ & Clinical & $\begin{array}{l}N=20 \\
\text { All RA }\end{array}$ & $\begin{array}{l}\text { Patients selected by } \\
\text { rheumatologists as successful } \\
\text { in dealing with implications of } \\
\text { RA }\end{array}$ & $\begin{array}{l}\text { Age: } \\
\text { Women 13/20 } \\
(65 \%)\end{array}$ & $\begin{array}{l}\text { To investigation the } \\
\text { relationship between social } \\
\text { support and acceptance in } \\
\text { patients with RA }\end{array}$ & $\begin{array}{l}\text { Qualitative } \\
\text { Semi- } \\
\text { structured } \\
\text { interviews }\end{array}$ \\
\hline $\begin{array}{l}\text { Kristiansen } \\
(2012)[43] \\
\text { Denmark } \\
(\text { High } \\
\left.\text { income }{ }^{a}\right)\end{array}$ & Clinical & $\begin{array}{l}N=32 \\
\text { All RA }\end{array}$ & Outpatient clinics (Multicentre) & $\begin{array}{l}\text { Age (mean): } \\
58.2 \text { years } \\
\text { Women } 19 / 32 \\
(59.4 \%)\end{array}$ & $\begin{array}{l}\text { To explore effects of RA on } \\
\text { everyday life }\end{array}$ & $\begin{array}{l}\text { Qualitative } \\
\text { Focus groups }\end{array}$ \\
\hline $\begin{array}{l}\text { Laidmae } \\
\text { (2009) [44] } \\
\text { Estonia (High }_{\text {income }}{ }^{\mathrm{a}} \text { ) }\end{array}$ & Clinical & $\begin{array}{l}N=808 \\
\text { All RA }\end{array}$ & $\begin{array}{l}\text { Hospitals and health centres } \\
\text { (multicentre) }\end{array}$ & $\begin{array}{l}\text { Age: } 66 \% \text { ( } n= \\
533) \text { over } 56 \\
\text { years } \\
\text { [No range or } \\
\text { mean age given] } \\
\text { Female: } 687 \\
(85 \%)\end{array}$ & $\begin{array}{l}\text { To investigate the impact of } \\
\text { RA on quality of life, and the } \\
\text { role of support and } \\
\text { assistance from family } \\
\text { members/acquaintances }\end{array}$ & $\begin{array}{l}\text { Quantitative: } \\
\text { cross-sectional } \\
\text { study, using } \\
\text { self- } \\
\text { administered } \\
\text { questionnaire }\end{array}$ \\
\hline $\begin{array}{l}\text { Lempp (2006) } \\
\text { [45] } \\
\text { England (High } \\
\left.\text { income }^{a}\right)\end{array}$ & Clinical & $\begin{array}{l}N=26 \\
\text { All RA }\end{array}$ & $\begin{array}{l}\text { Rheumatology outpatient } \\
\text { clinics (multicentre) }\end{array}$ & $\begin{array}{l}\text { Age(mean): } 56 \\
\text { years } \\
\text { Female 22/ } \\
\text { 26(84.6\%) }\end{array}$ & $\begin{array}{l}\text { To understand personal } \\
\text { experiences of living with } \\
\text { RA, and impact of RA upon } \\
\text { patients' lives }\end{array}$ & $\begin{array}{l}\text { Qualitative } \\
\text { Semi- } \\
\text { structured } \\
\text { interviews }\end{array}$ \\
\hline $\begin{array}{l}\text { Neville (1999) } \\
\text { [31] } \\
\text { Canada (High } \\
\text { incomea }^{a} \text { ) }\end{array}$ & Clinical & $\begin{array}{l}N=197 \\
\text { - RA: } 57 \\
\text { - SLE: } 27 \\
\text { - OA: } 41 \\
\text { - Back Pain: } 55 \\
\text { - Systemic } \\
\text { sclerosis: } 17\end{array}$ & $\begin{array}{l}\text { Rheumatology outpatient } \\
\text { clinic (public and private, } \\
\text { multicentre) }\end{array}$ & $\begin{array}{l}\text { Age (mean): } 60 \\
+/-15 \text { years } \\
\text { Female 164/197 } \\
(83.2 \%)\end{array}$ & $\begin{array}{l}\text { To identify concerns \& } \\
\text { learning interests of arthritis } \\
\text { patients }\end{array}$ & $\begin{array}{l}\text { Quantitative } \\
\text { Descriptive } \\
\text { cross-sectional } \\
\text { self- } \\
\text { administered } \\
\text { questionnaires }\end{array}$ \\
\hline $\begin{array}{l}\text { Sato (2008) } \\
{[46]} \\
\text { Japan (High } \\
\text { income })\end{array}$ & Clinical & $\begin{array}{l}N=364 \\
\text { All RA }\end{array}$ & $\begin{array}{l}\text { Commercial healthcare } \\
\text { database services (patient } \\
\text { records) }\end{array}$ & $\begin{array}{l}\text { Age (mean): } 45.5 \\
+/-8.4 \text { years } \\
\text { Female 288/364 } \\
(79.1 \%)\end{array}$ & $\begin{array}{l}\text { To describe the nature of } \\
\text { benefit finding in } \\
\text { rheumatoid arthritis } \\
\text { including predictive social } \\
\text { factors and impact on } \\
\text { mental health }\end{array}$ & $\begin{array}{l}\text { Quantitative } \\
\text { Questionnaires }\end{array}$ \\
\hline $\begin{array}{l}\text { Strand (2015) } \\
\text { [47] } \\
\text { USA/Europe } \\
\text { (high } \\
\text { income }\end{array}$ & Clinical & $\begin{array}{l}N=1958 \\
\text { All RA }\end{array}$ & Internet survey & $\begin{array}{l}\text { Age (mean): } 46 \\
+/-10.4 \text { years } \\
\text { Female } 100 \%\end{array}$ & $\begin{array}{l}\text { To identify effects of RA and } \\
\text { the impact of goal-setting } \\
\text { strategies }\end{array}$ & $\begin{array}{l}\text { Quantitative } \\
\text { Questionnaires } \\
\text { (2 different } \\
\text { surveys) }\end{array}$ \\
\hline $\begin{array}{l}\text { Sverker (2015) } \\
{[48]} \\
\text { Sweden (High } \\
\left.\text { income }^{a}\right)\end{array}$ & $\begin{array}{l}\text { Clinical (ACR } \\
\text { criteria) }\end{array}$ & $\begin{array}{l}N=48 \\
\text { All RA }\end{array}$ & $\begin{array}{l}\text { Rheumatology outpatient } \\
\text { clinics }\end{array}$ & $\begin{array}{l}\text { Age 20-45: } n=9 \\
\text { Age 46-55: } n= \\
11 \\
\text { Age } 56-60 n= \\
18 \\
\text { Age 61-63: } n= \\
10 \\
\text { Female } 71 \%\end{array}$ & $\begin{array}{l}\text { To explore the experience of } \\
\text { early RA in everyday life }\end{array}$ & $\begin{array}{l}\text { Qualitative } \\
\text { Semi- } \\
\text { structured } \\
\text { interviews }\end{array}$ \\
\hline $\begin{array}{l}\text { Thomas } \\
(2019)[49] \\
\text { UK }(H i g h \\
\left.\text { income }{ }^{a}\right)\end{array}$ & Clinical & $N=15$ & $\begin{array}{l}\text { Rheumatology outpatient } \\
\text { clinic }\end{array}$ & $\begin{array}{l}\text { Age (mean): } 56 \\
\text { years (range } 29- \\
80 \text { years) } \\
\text { Female: } 12 / 15 \\
(80 \%)\end{array}$ & $\begin{array}{l}\text { To explore the perspectives, } \\
\text { experiences and strategies } \\
\text { employed by people with } \\
\text { RA who engage in regular } \\
\text { physical activity. }\end{array}$ & $\begin{array}{l}\text { Qualitative } \\
\text { Semi- } \\
\text { structured } \\
\text { interviews }\end{array}$ \\
\hline $\begin{array}{l}\text { Van der Meer } \\
(2011)[50] \\
\text { Netherlands } \\
\text { (High }\end{array}$ & Clinical & $\begin{array}{l}N=14 \\
\text { All RA }\end{array}$ & $\begin{array}{l}\text { Rheumatology outpatient } \\
\text { clinic }\end{array}$ & $\begin{array}{l}\text { Age }(\text { mean): } 47 \\
+/-2.9 \text { years } \\
\text { Female } 12 / 14 \\
(85.7 \%)\end{array}$ & $\begin{array}{l}\text { To investigate patient } \\
\text { experiences and needs in } \\
\text { work participation of people } \\
\text { with RA treated with anti- }\end{array}$ & $\begin{array}{l}\text { Qualitative } \\
\text { In-depth } \\
\text { interviews }\end{array}$ \\
\hline
\end{tabular}


Table 1 Included manuscripts relating to scoping review of consumer perceived other service needs related to osteoarthritis and inflammatory arthritis, including rheumatoid arthritis and other inflammatory conditions (Continued)

\begin{tabular}{|c|c|c|c|c|c|c|}
\hline income $\left.e^{a}\right)$ & & & & & $\begin{array}{l}\text { tumour necrosis factor (TNF) } \\
\text { therapy }\end{array}$ & \\
\hline $\begin{array}{l}\text { Wollenhaupt } \\
\text { (2013) [51] } \\
\text { Germany } \\
\text { (High } \\
\text { income }{ }^{a} \text { ) }\end{array}$ & Not specified & $\begin{array}{l}N=318 \\
\text { All RA }\end{array}$ & $\begin{array}{l}\text { Members of a German } \\
\text { association for rheumatic } \\
\text { diseases }\end{array}$ & $\begin{array}{l}\text { Age: } 60+63.5 \% \\
\text { Female } 83.3 \%\end{array}$ & $\begin{array}{l}\text { To assess quality of life as } \\
\text { well as perceived needs and } \\
\text { expectations for treatment } \\
\text { and support }\end{array}$ & $\begin{array}{l}\text { Quantitative } \\
\text { Questionnaires }\end{array}$ \\
\hline
\end{tabular}

${ }^{a}$ Income stratification according to World Bank Country and Lending Groups 2019 Fiscal report based on Gross National Income per Capita [52]

Nine OA studies (Table 2) [22-27, 30-32] and 17 IA studies (Table 3) [33-37, 41-44, 46-48, 51, 53] identified issues relating to social participation and connectedness.

Of the OA studies, mobility restriction and lack of suitable transportation significantly limited social participation $[24,25]$. Inability to participant in group activities restricted social contact [24], with family activities limited to avoid excluding family members [22]. Hand OA limited participants' ability to conform to social norms [30] due to functional constraints [27], which could cause embarrassment [27]. Some participants worried about the appearance of their hands [30]. Strong social support was linked to better health perceptions [26]. Caring for grandchildren could be difficult, including limited ability to lift toddlers and change nappies [24, 25]. Both doctors and support networks sometimes failed to recognise OA as a "real" disease, contributing to communication difficulties [23]. People were frustrated by a lack of community support, and underestimation of pain and suffering [32]. Media coverage of, and research into OA was perceived as inadequate [23]. One study suggested that only $20 \%$ of people with OA desired access to support groups [31].

In people with IA, social connectedness [31, 35, 39$41,43,44,48,53$ ] and peer support was critical. Loneliness and withdrawal were common [43, 44], especially for those living alone [44]. IA put strain on personal relationships [46] particularly ability to provide care for others [48], and relationships with partners including sexual function and intimacy [33, 46, 47]. Some experienced lowered mood due to pain [36]. Participants were desperate for support from family and often felt "alone" [37], needing to trust others to accept proffered support [35]. This was exacerbated by limitation of social activities $[36,51]$ and losing employment $[43,45]$. Barriers to social connectedness included perceived lack of understanding [33, 35, 36, 43], financial difficulties, mobility problems and fear of falling victim to crime [44]. Overall, participants felt less able to participate in social events, community life and relationships [33, 36, 48]. Assistive devices made some participants feel embarrassed or "weird" [23]. Participants with PsA and foot involvement felt demoralised or stigmatised by their appearance and need for specialised footwear [36]. Participants were interested in self-help groups [31, 36], which helped with coping and self-management through support, recognition and legitimisation of personal experiences and problems [39, 43]. These groups facilitated participants building new relationships [39, 43], and proving that normality is possible [43]. Having relatives with the same condition helped with coping [36]. Peer groups supporting physical activity were desirable [40].

\section{3) Financial needs and security}

Three OA studies (Table 2) [25, 27, 28] and three IA studies (Table 3) [31, 44, 46] investigated financial needs and security.

OA studies focussed on the cost of health-seeking behaviour [25] and need for financial security. Work capacity was limited by disease [27, 28].

In IA, financial stress was common [31, 44]. Income protection was accessed by $32 \%$ [46]. Participants from Estonia described financial concerns limiting their access to basic needs including food, running water and heating, as well as sociocultural experiences [44].

\section{4) Occupational needs}

Four OA articles (Table 2) $[24,25,28,30]$ and 13 IA studies (Table 3) [31, 33, 36, 38, 39, 43-47, 50, 51] identified needs related to work.

People with OA wanted a flexible workplace [24, 25, 28] to facilitate work retention [24]. Flexibility entailed the need for regular breaks and environmental modification, although aides and environmental modifications were often not available [24]. Failure of these supports could require people to change employment $[24,25,28]$. Hand OA could limit work productivity [30].

IA often affected ability to work, particularly productivity, self-confidence, career progression or salary [33, $36,46,47]$. However, people with IA valued working to maintain a normal life $[31,39,43-45,50]$, financial security [39, 44], self-esteem, identity [43] and social networks [43]. Disability pensions were seen as a "last resort" [39]. At least 34\% percent experienced difficulties at work, increased absenteeism in $11 \%$ and premature 
Table 2 Results of scoping review of consumer perceived other service needs related to osteoarthritis

\begin{tabular}{|c|c|}
\hline Author, Year & Results \\
\hline \multicolumn{2}{|c|}{ ACTIVITIES OF LIVING BOTH INSIDE AND OUTSIDE THE HOME } \\
\hline Ackerman (2013) [21] & $\begin{array}{l}\text { - Cannot get out of house without assistance } \\
\text { - Difficulties walking due to OA, limited mobility }\end{array}$ \\
\hline Al-Taiar (2013) [22] & $\begin{array}{l}\text { - Inability to do household chores, mobility limitation } \\
\text { - Many participants have domestic helpers } \\
\text { - "Failure" to fulfil obligation to take care of the family despite their pain/mobility limitation; feeling helpless/less valuable }\end{array}$ \\
\hline Bergsten (2011) [35] & $\begin{array}{l}\text { - Need for support from family and friends when doing household duties, personal care or everyday activities } \\
\text { - Struggling to accept help from others; wanting to do more }\end{array}$ \\
\hline $\begin{array}{l}\text { Bukhave \& Huniche } \\
\text { (2014) [24] }\end{array}$ & $\begin{array}{l}\text { - Difficulty handling small objects e.g. cutlery, glasses, gadgets, chargers, plugs and devices for connecting gadgets to } \\
\text { power supplies, computers, cell phones (especially if buttons too small), money and payment systems (credit cards } \\
\text { easier) } \\
\text { - Difficulties determined by design/operation of the actual gadget } \\
\text { - Dependency on help from others (partners most important providers of support), particularly with respect to the } \\
\text { performing of household chores and self-care (grooming, hair dryers, buttons, tying shoes) } \\
\text { - Singles with small networks experienced huge challenges } \\
\text { - Need for external help at time; expensive } \\
\text { - Importance of good grooming to participants } \\
\text { - Special equipment, assistive devices or orthoses can improve performance; e.g. self-adapted knife }\end{array}$ \\
\hline Chan (2011) [25] & $\begin{array}{l}\text { - Reliant on support from the family or paid supports } \\
\text { - Need to be accompanied by others when going out }\end{array}$ \\
\hline Hill (2010) [27] & $\begin{array}{l}\text { - Limited function in day-to-day activities including self-care activities } 66 \% \text { (cutting fingernails, drying after showering, } \\
\text { toileting) } \\
\text { - Difficulties with opening packaging, peeling fruit and vegetables, cutting } \\
\text { - Limitation of hobbies/past times } \\
\text { - Gender differences: men reported difficulties with manual work and particular hobbies (fishing, car mechanics), women } \\
\text { reported difficulties with home-making tasks (housework, cooking) } \\
\text { - Feelings of "frustration" at inability to do things in } 55 \% \text {; may lead to depression } \\
\text { - Transitioning from normal function "taking it for granted"; loss of identify/sense of self because of being unable to do } \\
\text { things previously done } \\
\text { - Inability to conform to social norms due to functional constraints causing embarrassment/self-consciousness } \\
\text { - Utility of assistive devices/adaptations to improve function and independence }\end{array}$ \\
\hline Kjeken (2013) [29] & $\begin{array}{l}\text { - Strategies to improve function in daily activities: } \\
\text { - Assistive devices: opening packaging, cutting food } \\
\text { - Adapting tools/materials/working techniques: e.g. facilitating lifting/carrying, housework, opening packaging } \\
\text { - Practice activity pacing: planning daily activities and rest breaks to enable task completion } \\
\text { - Stop or avoiding certain activities } \\
\text { - Importance of positive thinking in completing tasks: focussing on what you can do, not pain or limitation, } \\
\text { perseverance } \\
\text { - Communication: ask/apply/pay for help, telling people about needs/problems }\end{array}$ \\
\hline Neville (1999) [31] & - $75 \%$ report needing more help carrying out daily tasks \\
\hline Tanimura (2011) [32] & $\begin{array}{l}\text { - Restriction of daily activities } 70.5 \% \\
\text { - Taking more time to complete daily activities } 66 \% \\
\text { - Difficulty sitting on traditional straw matting ("tatami") } 94.7 \% \text {, sitting up/squatting down } 93.7 \% \text {, going up or downstairs } \\
61.2 \% \text {, sitting in same position for extended periods } 93.1 \% \text {, carrying heavy objects ( } 88.4 \%)\end{array}$ \\
\hline
\end{tabular}

\section{SOCIAL PARTICIPATION NEEDS}

Al-Taiar, (2013) [22] • Mobility restriction affects social life (including attending events like weddings)

- Whole family affected rather than leave participant at home alone; especially young children/teenagers requiring supervision

Baumann et al., (2007)

- Emotional distress as well as physical limitations; difficulty communicating struggle with family or doctors

- Unrecognised disability; lack of recognition by family and friends (not seeing OA as a "real" disease), community (e.g. access to disability permits), lack of OA-related research and media coverage

- Importance of support from others with the same condition; "It's so nice to feel you are not the only one suffering"

Bukhave \& Huniche, - Limited participation in activities requiring withdrawal from group activities (e.g. skiing, canoeing, dancing, woodwork (2014) [24] and holding dinner parties) resulting in reduction of social network

- Difficulty caring for grandchildren, including lifting and carrying children

Chan, (2011) [25] - May have to cut down or abstain from social activities

- Often limited choice of social activities depending on available transportation and walking distance

- Difficulty playing with/looking after grandchildren

Ilori (2016) [26]

- Social support most commonly provided by children (68.8\%)

- Perceptions of "good health" significantly more common in those with strong support from family (69.9\%) and friends 
Table 2 Results of scoping review of consumer perceived other service needs related to osteoarthritis (Continued)

\begin{tabular}{|c|c|}
\hline Author, Year & Results \\
\hline & $\begin{array}{l}\text { (71.6\%) cf. those with weak support from family }(47.1 \%) \text { or friends }(59.6 \%) \\
\text { - High functional health significantly more common in those with strong support from family, friends and significant } \\
\text { other than those with weak support. }\end{array}$ \\
\hline Hill (2010) [27] & $\begin{array}{l}\text { - Unable to conform to social norms due to functional constraints, causing feelings of embarrassment } \\
\text { - Comparison with others made people more aware of disability, but sometimes reminded people that others were } \\
\text { worse off }\end{array}$ \\
\hline Leung et al. (2019) [30] & $\begin{array}{l}\text { - Hand OA had significant impact on ability to participate in social roles in } 33.3 \% \text {, emotional health and mood in } 28.9 \% \text {, } \\
\text { ability to participate in social ability in } 31.1 \% \text { and appearance of hands/self-image in } 37.8 \%\end{array}$ \\
\hline Neville (1999) [31] & - $20 \%$ of OA patients interested in a self-help group \\
\hline Tanimura (2011) [32] & - Lack of recognition of knee pain by others $58.2 \%$ \\
\hline \multicolumn{2}{|c|}{ FINANCIAL NEEDS AND SECURITY } \\
\hline Chan (2011) [25] & - Monetary costs of treatments affect health seeking behaviour \\
\hline Hill (2010) [27] & $\begin{array}{l}\text { - 2/29 forced to retire from work due to hand problems; significant financial implications of giving up work } \\
\text { - Struggling to handle money and write cheques due to hand OA }\end{array}$ \\
\hline Kao (2014) [28] & - Reduction of work affecting household income (87.5\% labourers); $61.5 \%$ were the main income earner \\
\hline \multicolumn{2}{|l|}{ OCCUPATIONAL NEEDS } \\
\hline $\begin{array}{l}\text { Bukhave \& Huniche } \\
\text { (2014) [24] }\end{array}$ & $\begin{array}{l}\text { - Struggle to keep working until retirement age } \\
\text { - Some had option for flexibility in arrangements with employers; depends on individual work demands, may need to } \\
\text { change to a job where demands match hand function } \\
\text { - Often lack of adaptation of work environment and technical aids, and lack of knowledge concerning workplace } \\
\text { adaptations and technical aids that could have been offered by the employer } \\
\text { - Flexibility important }\end{array}$ \\
\hline Chan (2011) [25] & $\begin{array}{l}\text { - Impacts on work life included: tiring easily, feeling inconvenient, less efficient, need to take sick leave, need to quit job, } \\
\text { fewer business trips / do less business } \\
\text { - Some forced to change job/resign/early retirement }\end{array}$ \\
\hline Kao (2014) [28] & $\begin{array}{l}\text { - Need to reduce work, adjust work content and exchange work } \\
\text { - Limitation of work due to pain }\end{array}$ \\
\hline Leung et al. (2019) [30] & - Hand OA had a significant impact on work productivity in $33.3 \%$ \\
\hline
\end{tabular}

\title{
EXERCISE AND LEISURE-RELATED NEEDS
}

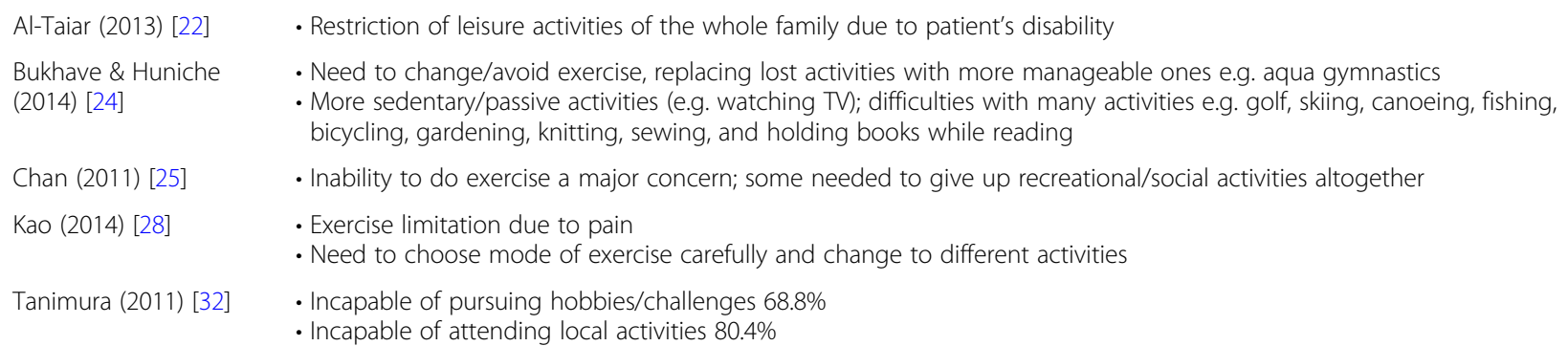

\section{TRANSPORT NEEDS AND ENVIRONMENTAL MODIFICATION}

\author{
Ackerman et al. (2013) • Transport difficulties in 22\% \\ [21] \\ Bukhave \& Huniche - Difficulty with handling the shift, holding on to the steering wheel, opening doors and the boot and handling the \\ (2014) [24] \\ petrol cap of a car \\ - Difficulty riding a bike e.g. hand brakes, shifting gears, lamps and locks \\ - Difficulty with public transport e.g. holding on to straps or poles during exacerbating pain/other symptoms \\ Chan (2011) [25] - Difficulty going out, particularly taking public transport; worsens with disease progression \\ - Lack of suitable public transport facilities \\ - Use of walking sticks \\ Kao (2014) [28] - Did not enjoy travelling, especially getting in and out of the car \\ - Pain an inconvenience e.g. climbing stairs, needing to look for seated toilets \\ - Need to use analgesia prior to outings
}


Table 3 Results of scoping review of consumer perceived other service needs related to inflammatory arthritis

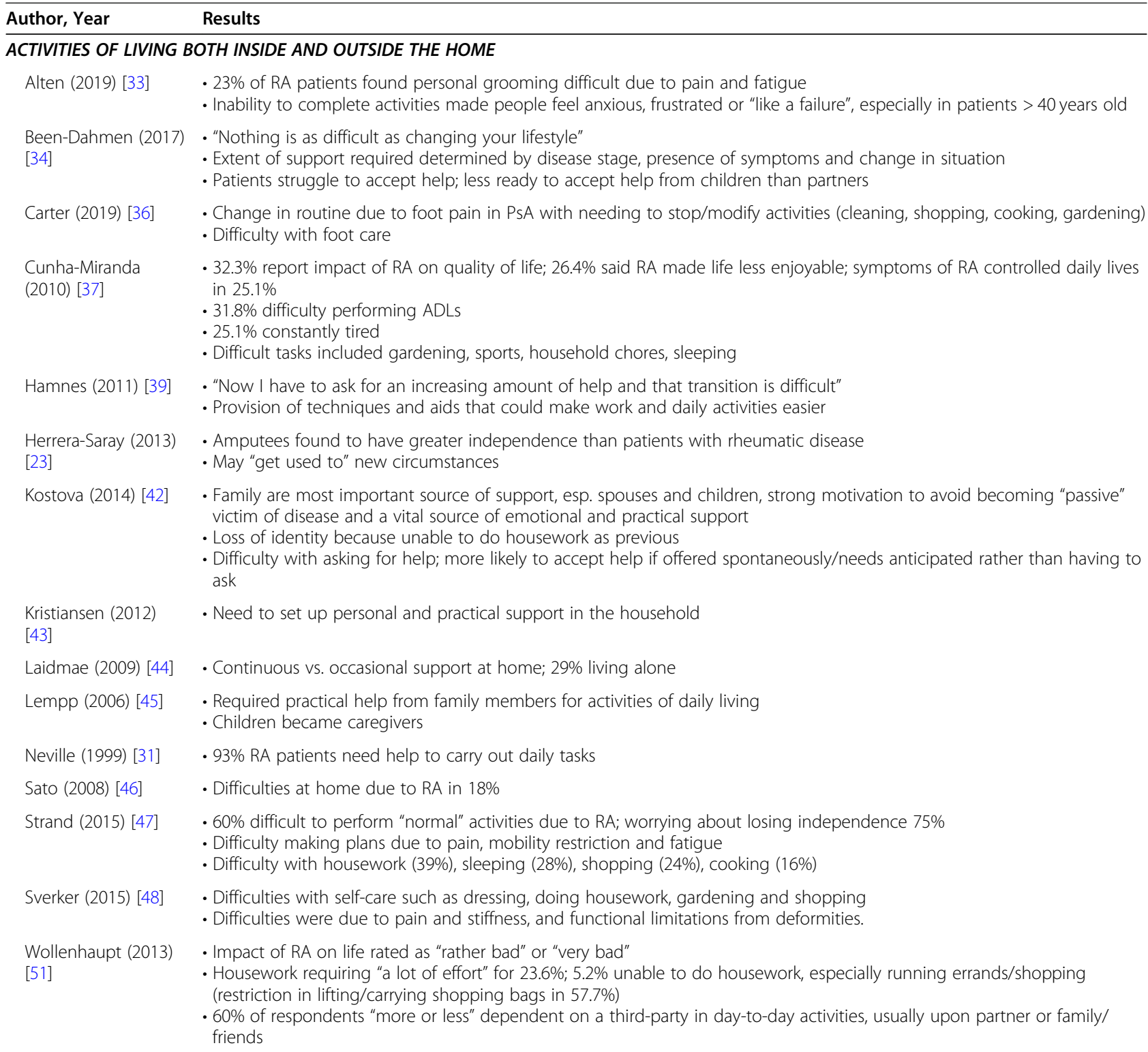

\section{SOCIAL PARTICIPATION NEEDS}

Alten (2019) [33] • 35-39\% of people reported difficulty with others understanding their disease

- Negative impact on relationship with spouse or partner, including sex life and intimacy

- Negative impact on inclusion in family and social events

- Better understanding from others in those with a partner or children; $43 \%$ wished for better understanding of disease impact from others

Been-Dahmen (2017) • Trusting relationship with professionals, relatives and fellow patients

[34] - Emotional support required from relatives; however, they did not always recognise emotional issues. Partners more capable than children.

- Most did not need support from fellow patients; some appreciated shared experiences. Most not interested in formal group meetings.

Bergsten (2011) [35] • Need for support from friends and family, as well as healthcare professionals, but patients need to trust/accept support offered

- Need for friends and relatives to understand difficulties faced/problems created by disease

Carter(2019) [36] • Spending time with family and friends disrupted due to foot symptoms and functional limitations

- Lowered mood due to preoccupation with pain; reliance on family members for support

- Better understanding/empathy from those with affected family members; some found benefit from support groups

- Patients with PSA and foot problems conscious of change to physical appearance and footwear restrictions; demoralised 
Table 3 Results of scoping review of consumer perceived other service needs related to inflammatory arthritis (Continued)

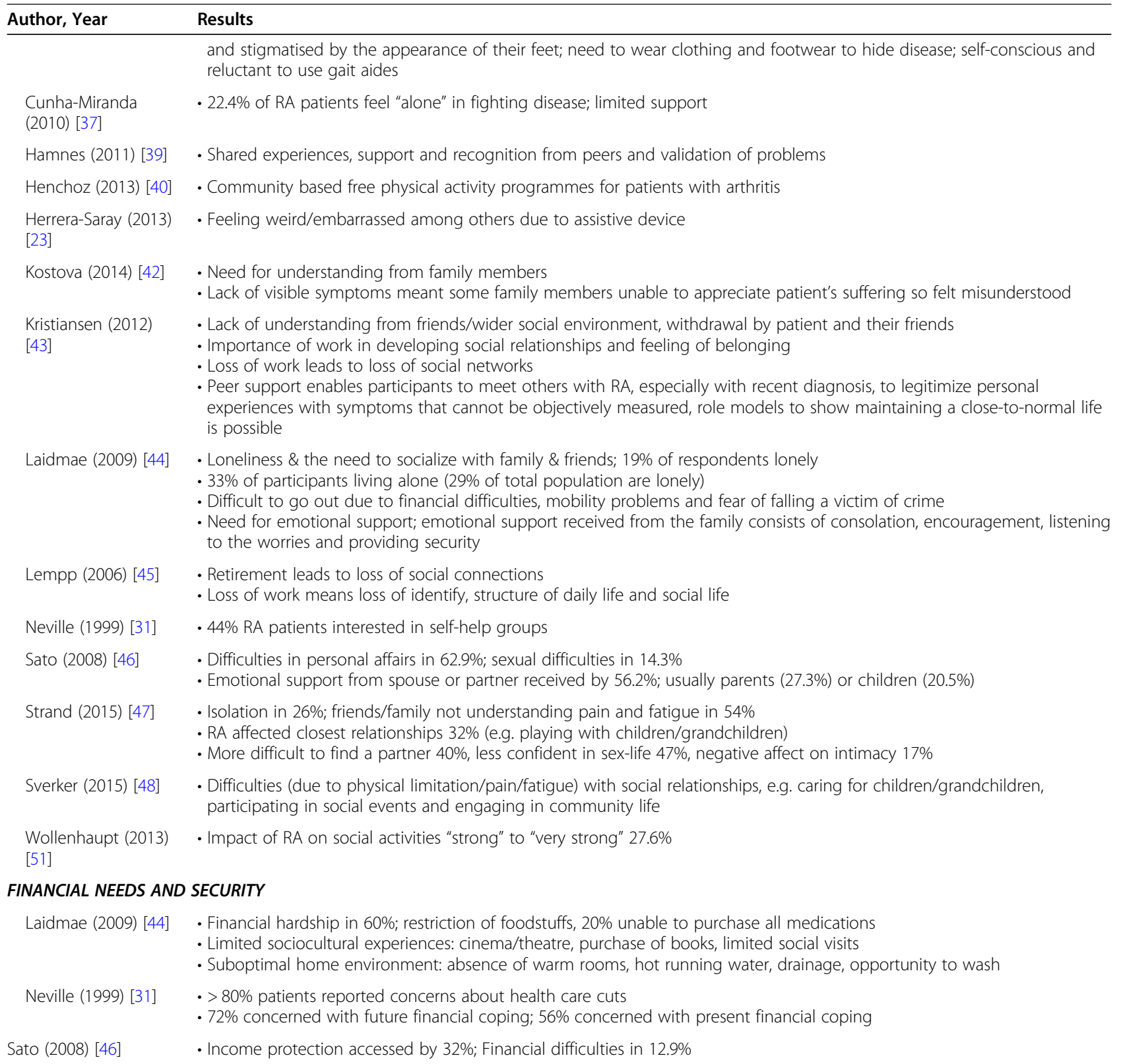

\section{OCCUPATIONAL NEEDS}

Alten (2019) [33] 95\% of participants reported leave, retirement or lack of career progression since RA diagnosis; 18\% forced to retire and 23\% slow career progression

- $31 \%$ inadequate physical accommodations at work, 36\% inadequate emotional accommodations

- Barriers to work include difficulty with hand function (44\%), pain (43\%), unpredictable state of health (34\%)

Carter (2019) [36] - Foot-related disability contributed to loss of work, or difficulty performing jobs due to foot pain and stiffness

- Impact of modified footwear on job roles e.g. unable to wear dress shoes or safety boots

Cunha-Miranda $\quad$ - RA affected ability to work: $24.7 \%$

(2010) [37] - Absence from work due to illness: 21.6\% (mean duration of absence 16-17 days)

Giacomelli (2015) 34\% reported difficulties at work; increased work absenteeism in 11, 7.9\% retired

[38]

Hamnes (2011) [39] • Need to continue to work, important to avoid disability pension (last resort)

- Wanted to know work-related rights and rights related to social security

Kristiansen (2012) Need to continue work (with or without special conditions); this helped to maintain normal life and sense of normality; need for [43] support to clarify work capacity 
Table 3 Results of scoping review of consumer perceived other service needs related to inflammatory arthritis (Continued)

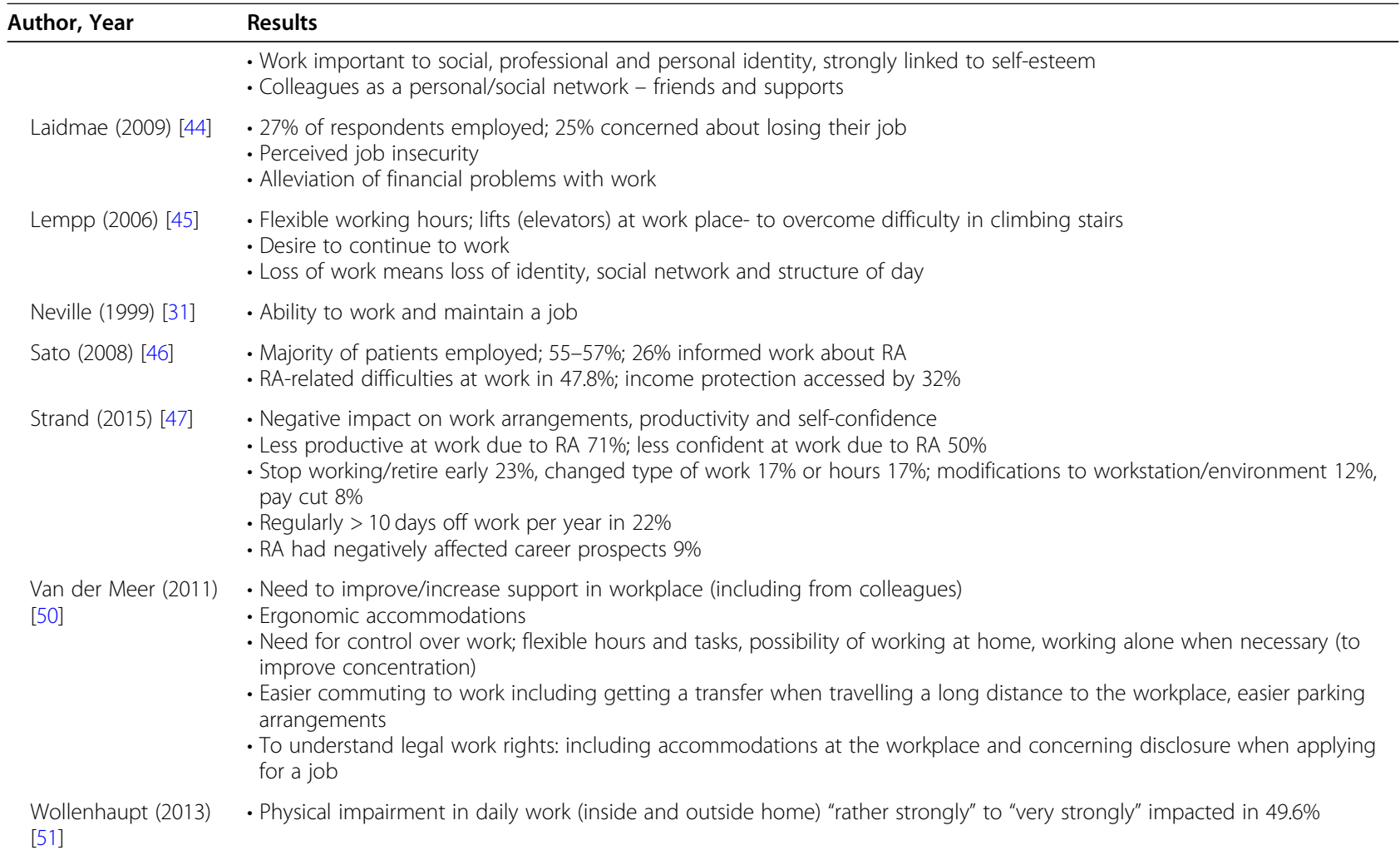

\section{EXERCISE AND LEISURE-RELATED NEEDS}

Been-Dahmen (2017) Empowered by information about type and necessity of physical exercise, as well as seeing other patients exercising [34]

Bergsten (2011) [35] • Unable to do particular physical activities

Carter (2019) [36] • Difficulty with walking especially on uneven ground in those with PSA and foot involvement

Cunha-Miranda $\quad$ - Less able to do sports

(2010) [37]

Henchoz (2013) [40] Physical, psychological, functional and social benefits to exercise; arthritis specific barriers e.g. loss of function, pain, stiffness, concern of peers

- No programs/consideration for those with arthritis

- Non- arthritis specific barriers eg scheduling, cost, lack of time, peers do not exercise, carer responsibilities, etc

Strand (2015) [47] • Adverse effect of RA on social, family and leisure activities

- Limited enjoyable activities (42\%) and spontaneity (57\%), keeping fit/playing spots (46\%), gardening (39\%), outdoor activities (33\%)

- Favourite hobby painful in $31 \%$

Thomas (2019) [49] - Need for physical activity as a key part of managing RA; symptoms may help to motivate people to be physically active - Options where physical activity also had a social element, as a mode of transportation, dog walking all popular forms of activity

- Some hesitation about general group activity classes; concern re: being unable to keep up or lack of understanding of RA

\section{TRANSPORT NEEDS AND ENVIRONMENTAL MODIFICATION}

Herrera-Saray (2013) • Architectural barriers in the home, the workplace and/or outdoors

[23] - Lack of design standards for persons with disabilities, e.g. ramps, parking spaces and ample space for movement

Henchoz (2013) [40] • Environmental modifications favourable for physical activity: availability of facilities free of charge, maintenance of pavements, streetlights

Laidmae, (2009) [44] • Fear of falling victim of crime (16\%); perceived increased risk due to physical impairment and poor health - Transport needs

Strand (2015) [47] • Difficulty with driving in 17\%

Wollenhaupt (2013) • Unable to drive a car 6.9\% [51] 
retirement in $8-18 \%[33,38]$ with $25 \%$ worrying about job losses [44]. The need for modified footwear impacts on job roles in those with PsA and foot disease (e.g. unable to wear dress shoes or safety boots) [36]. Factors improving work retention included physical ability [51], travel arrangements (parking, working from home) [50], flexibility of hours and conditions $[45,50]$ and modifications in the workplace [45, 50]. Participants wanted more information about their work-related rights [39, 50].

5) Exercise and leisure related needs

Five OA studies (Table 2) [22, 24, 25, 28] identified barriers to exercise and leisure participation. Disease progression meant people needed to modify or swap exercise/leisure activities [24]. People worried about their inability to exercise [25], especially pain, inability to continue activities previously enjoyed [28, 32], missing out on activities with others [22] and needing to engage in more sedentary activities [24].

Seven studies investigated needs and attitudes of people with IA to exercise and leisure activities (Table 3) [34-37, 40, 47, 49]. Participants felt empowered by information about type and necessity of physical exercise [34]. In RA, physical, psychological, functional and social benefits from exercise were identified $[35,40]$; exercise was identified as a critical part of self-management [49]. However, RA limited mobility and caused pain, restricting participation in specific exercises/sports [37, 40], with some activities wholly inaccessible [35]. Some participants found RA symptoms to be a motivator to be active [49]. Social, family and leisure activities were affected, limiting sports, fitness, hobbies and spontaneity [47]. Participants preferred options where physical activity served an additional purpose e.g. social contact, transportation, dog walking [49]. Participants identified a lack of RA-specific exercise programs, with exercise programs and instructors failing to consider limitations imposed by arthritis [40] or hesitation about joining general exercise classes due to being unable to keep up or lack of understanding [49]. Patients with PsA and foot disease struggled with walking, particularly on uneven ground [36].

6) Transport needs and environmental modification

Four OA studies (Table 2) [21, 24, 25, 28] investigated needs related to transport. More than $20 \%$ of people experienced difficulties in one study [21]. Services to increase accessibility to public transport may improve people's ability to socialise [25]. In people with hand OA, supports were needed to facilitate opening doors, holding the steering wheel and using bicycle hand brakes
[24], with use of public transportation limited by their inability to hold straps/poles [24]. Travel required significant planning, including in getting in and out of cars, finding seated toilets in Taiwan and needing plan analgesia around going out [28].

Five IA studies (Table 3) [40, 41, 44, 47, 51] identified environmental limitations to moving about outside the home. These included lack of appropriate transport [44] and unsafe environment related to fear of falling related to environmental factors (uneven pavement, lack of ramps and lighting) $[40,41,44]$. Driving could be difficult $[47,51]$. Some participants worried about falling victim of crime due to frailty [44].

\section{Discussion}

This review demonstrates the pervasive impact of arthritis on peoples' lives, independent of aetiology. We have identified six key domains in which arthritis impacts life: daily living, social participation, financial security, occupation, exercise/leisure and transportation. All areas of need identified were common to OA and IA, illustrating that need appears to primarily be linked to symptoms common across musculoskeletal conditions, rather than aetiology or pathogenesis.

Non-healthcare needs related maintain daily functioning were identified by people with both OA and IA. Deficits lead to a sense of "failure", particularly being unable to do household chores or care for children [22]. The pervasiveness of this theme highlights the importance of supporting functional ability in both ADLs and the workplace. People with arthritis appear to need robust social support systems to assist them with ADLs [22], and targeted assistive devices (e.g. for cooking) to enable participants to complete tasks and feel "normal" [23, 24, 40].

Needs related to social functioning were similar in people with IA and OA. Loneliness, withdrawal $[24,25$, $43,44]$, and lack of understanding from family, friends and communities were troublesome [23, 43], but partly negated by peer support groups [23]. People with both IA and OA wanted means to reduce social isolation. In the wider literature, social connectedness and "diffuse social relationships" have been identified as crucial to psychological wellbeing [54]. An individual's health can be related to the strength of their social relationships, with participants in this review with "strong" social supports more likely to perceive good health [26]. Interestingly, in one study people with IA tended to be more interested (44\%) in peer support groups than those with OA (20\%) [31]. Further data are required regarding optimal delivery of peer support groups, particularly as selfmanagement education groups are limited in effectiveness for most clinical outcomes [55]. 
The need for work retention was critical to people with both OA and IA, for financial security and social connectedness [43]. Arthritis is associated with reduced work productivity, early retirement and reduced wealth [6]. Flexibility and environmental adaptations in the workplace facilitated work retention in people with IA and OA $[24,25]$. Maintaining employment is a key issue in people with RA, facilitated by both environmental adaptations and flexible work hours [56]. Use of modified schedules when required was associated with lower workplace activity limitation, fewer job disruptions and productivity losses [56]. Furthermore, loss of work or retirement exacerbated feelings of social isolation, highlighting the importance of employment in social connectedness and self-worth [43, 45]. Programs targeted to improve work retention in people with arthritis can reduce anxiety, improve mood and life satisfaction [57]. Given the importance of employment in financial wellbeing and social connectedness, further work is needed to identify contributors to work retention in people with arthritis and to support and educate employers and practitioners in providing these.

Both OA and IA had similar detrimental effects on physical and leisure activity participation. When activities couldn't be modified/replaced, participants were excluded from activities with family and friends [22, 40]. Arthritis patients need assistance from healthcare providers with arthritis-specific exercise programs [58], as well as information about benefits and safety of exercise [34]. People with OA frequently report mobility and pain as barriers to participation in exercise [59], despite high quality evidence for its therapeutic benefits in $\mathrm{OA}$ and IA [60]. Access to practitioners with skills and knowledge in behaviour change, pain science and appropriate exercise programs/facilities is important for people with arthritis.

Transportation needs for those with IA and OA had broader impacts on other areas of need. Difficulties with transportation exacerbate dependence on others [40, 41], unemployment [50] and social isolation [32]. Transportation is intimately linked with freedom and independence in older adults, and has a pervasive impact on life [61]. Further research is required to understand factors limiting transportation and improve uptake in people with arthritis.

We have identified some contrasts between the experiences of people with OA and IA. Those with OA identified a lack of acknowledgement and community support $[23,43]$. They felt OA and the resultant disability were underestimated with limited media coverage of, and research into, OA [23]. While participants with IA felt that sometimes their symptoms were underestimated [47], they did not report trivialisation of the disease itself. In the wider literature people with RA felt inadequate support and information were available, particularly in specific situations like pregnancy [62]. Public health campaigns could assist with educating the wider community about arthritis and its impact.

This study has limitations. Firstly, although these data highlight the impact of OA and IA, existing literature focusses on problems related to arthritis rather than evaluation of actual needs. Thus, due to a paucity of data, we have not directly questioned the "needs" of people with $\mathrm{OA}$ and IA. It remains unknown which services exist and meet current needs, and which are insufficient, an important gap in the literature. This is a focus of the World Health Organisation's Integrated Care for Older people approach. However, in line with the Gothenburg model of person-centred care, a key step in providers being able to deliver effective, person-centred care is to understand the experience of the person [63]. Accordingly, we believe summarising the literature regarding these issues is an important step towards addressing non-healthcare needs of people with arthritis, and enabling the delivery of effective person-centred care. Given the limited data, it is difficult to comment on whether non-healthcare needs differ according to country or social setting. Furthermore, as participants included mainly post-menopausal females, generalizability may be limited to other groups (particularly men, younger people, and those in low- and middle-income settings). Studies included modest sample sizes. Heterogeneity of data collected means different areas were investigated in each study; this provides limited triangulation and/or validation of any single conclusion. Overall studies were at moderate risk of bias, with higher risk of bias in data collection and recruitment. Finally, we did not perform inter-rater reliability for study selection.

This review has numerous strengths. A comprehensive scoping literature search was performed across four different databases. Many qualitative studies were included to enable deeper exploration of participants' nonhealthcare needs and perspectives. This search captured data from multiple levels of care, including communitybased populations, as well as a range of different disease stages. OA studies involved a range of joints.

\section{Conclusions}

Arthritis has a pervasive impact on different areas of life, regardless of disease aetiology. To patients, the similarities in functional impact far outweigh the differences in the disease pathogenesis. Whilst people with arthritis are acutely aware of their inability to perform tasks and perceived "failures", little work has been performed to identify the patients' perspective of non-healthcare needs to facilitate targeted service provision and provide holistic care. Future research is required to assess this, across a broader population and joint involvement, to identify 
whether there may be joint-specific non-healthcare needs. Improved characterization of the patients' perceived non-healthcare needs is necessary to provide relevant support and services for people with arthritis.

\section{ABBREVATIONS}

ADLs: Activities of daily living; IA: Inflammatory arthritis; OA: Osteoarthritis; PSA: Psoriatic arthritis; RA: Rheumatoid arthritis; SLE: Systemic lupus erythematosus

\section{Supplementary Information}

The online version contains supplementary material available at https://doi. org/10.1186/s12891-021-04190-z.

\section{Additional file 1.}

\section{Acknowledgements}

Nil additional.

\section{Authors' contributions}

JF, MS and AW contributed to conception and design of study, analysis and interpretation of data, drafting the article, revising it critically for important intellectual content and final approval of the version to be submitted. SY and SA contributed to analysis and interpretation of data, revising the article critically for important intellectual content and final approval of version to be submitted. LC contributed to acquisition, analysis and interpretation of data, revising the article critically for important intellectual content and final approval of the version to be submitted. KS contributed to conception and design of the study, drafting the article and final approval of the version to be submitted. FM and AMB contributed to conception/design of the study, interpretation of the data, revising the article critically for important intellectual content and final approval of the version to be submitted. All authors have read and approved the manuscript.

\section{Funding}

This work was supported by a partnership grant awarded by Musculoskeletal Australia. Musculoskeletal Australia contributed to the conception of the review question but they did not influence the review execution, results or interpretation. L.C. is the recipient of an Australian Postgraduate Award and Arthritis Foundation Scholarship. A.E.W is the recipient of the recipient of the Career Development Fellowship, Royal Australasian College of Physicians.

\section{Availability of data and materials}

All data generated or analysed during the current study are included in this published article and its supplementary files.

\section{Declarations}

Ethics approval and consent to participate

Ethics approval was not required as this systematic review did not involve data collection or patient recruitment.

\section{Consent for publication}

Not applicable.

\section{Competing interests}

The authors declare no conflicts of interest or competing interests.

\section{Author details}

'Department of Epidemiology and Preventative Medicine, School of Public Health and Preventative Medicine, Monash University, Melbourne, Victoria, Australia. ${ }^{2}$ Department of Community Medicine, Faculty of Medical Sciences, University of Sri Jayewardenepura, Gangodawila, Nugegoda, Sri Lanka.

${ }^{3}$ Monash University Library, Monash University, Melbourne, Victoria, Australia.

${ }^{4}$ Curtin School of Allied Health, Curtin University, Perth, Western, Australia.
Received: 4 December 2020 Accepted: 24 March 2021

Published online: 09 April 2021

\section{References}

1. Foundation A. Arthritis by numbers. 2018.

2. IHME. The global burden of disease study 2019. Seattle; 2019. [updated 2019; cited 2021]; Available from: http://ghdx.healthdata.org/gbd-results-too

3. Brand CA. The role of self-management in designing care for people with osteoarthritis of the hip and knee. Med J Aust. 2008;189(S10):S25-8. https:// doi.org/10.5694/j.1326-5377.2008.tb02206.x.

4. Kahlenberg JM, Fox DA. Advances in the medical treatment of rheumatoid arthritis. Hand Clin. 2011;27(1):11-20. https://doi.org/10.1016/j.hcl.2010.09. 002.

5. Chou L, Ranger TA, Peiris W, Cicuttini FM, Urquhart DM, Sullivan K, et al. Patients' perceived needs for medical services for non-specific low back pain: a systematic scoping review. PLoS One. 2018;13(11):e0204885. https:// doi.org/10.1371/journal.pone.0204885.

6. Schofield DJ, Callander EJ, Shrestha RN, Percival R, Kelly SJ, Passey ME. Labour force participation and the influence of having arthritis on financial status. Rheumatol Int. 2015;35(7):1175-81. https://doi.org/10.1007/s00296-01 5-3224-2.

7. Berkovic D, Ayton D, Briggs AM, Ackerman IN. "I would be more of a liability than an asset": navigating the workplace as a younger person with arthritis. J Occup Rehabil. 2020;30(1):125-34. https://doi.org/10.1007/s10926-01909853-2.

8. Restoux LJ, Dasariraju SR, Ackerman IN, Van Doornum S, Romero L, Briggs AM. Systematic review of the impact of inflammatory arthritis on intimate relationships and sexual function. Arthritis Care Res. 2020;72(1):41-62. https://doi.org/10.1002/acr.23857.

9. Luong ML, Cleveland RJ, Nyrop KA, Callahan LF. Social determinants and osteoarthritis outcomes. Aging Health. 2012;8(4):413-37. https://doi.org/1 0.2217/ahe.12.43.

10. Tricco AC, Lillie E, Zarin W, O'Brien KK, Colquhoun H, Levac D, et al. Prisma extension for scoping reviews (prisma-scr): checklist and explanation. Ann Intern Med. 2018:169(7):467-73. https://doi.org/10.7326/M18-0850.

11. Wluka A, Chou L, Briggs AM, Cicuttini FM. Understanding the needs of consumers with musculoskeletal conditions: consumers' perceived needs of health information, health services and other non-medical services: a systematic scoping review; 2016.

12. Asadi-Lari M, Tamburini M, Gray D. Patients' needs, satisfaction, and health related quality of life: towards a comprehensive model. Health Qual Life Outcomes. 2004;2(1):32. https://doi.org/10.1186/1477-7525-2-32.

13. Segan JD, Briggs AM, Chou L, Connelly KL, Seneviwickrama M, Sullivan K, et al. Patient-perceived health service needs in inflammatory arthritis: a systematic scoping review. Semin Arthritis Rheum. 2018;47(6):765-77. https://doi.org/10.1016/j.semarthrit.2017.10.019.

14. Walsh D, Downe S. Meta-synthesis method for qualitative research: a literature review. J Adv Nurs. 2005;50(2):204-11. https://doi.org/10.1111/j.13 65-2648.2005.03380.x.

15. Hannes K, Macaitis K. A move to more systematic and transparent approaches in qualitative evidence synthesis: update on a review of published papers. Qual Res. 2012;12(4):402-42. https://doi.org/10.1177/14 68794111432992.

16. Campbell R, Pound P, Morgan M, Daker-White G, Britten N, Pill R, et al. Evaluating meta-ethnography: systematic analysis and synthesis of qualitative research. Health Technol Assess. 2011:15:1-164.

17. Noblit G, Hare R. Meta-ethnography: synthesizing qualitative studies; 1988. https://doi.org/10.4135/9781412985000.

18. France EF, Uny I, Ring N, Turley RL, Maxwell M, Duncan EAS, et al. A methodological systematic review of meta-ethnography conduct to articulate the complex analytical phases. BMC Med Res Methodol. 2019; 19(1):35. https://doi.org/10.1186/s12874-019-0670-7.

19. (CASP) CSAP. Casp qualitative checklist: Oxford Centre for Triple Value Healthcare 2014 Contract No.: Document Number.

20. Hoy D, Brooks P, Woolf A, Blyth F, March L, Bain C, et al. Assessing risk of bias in prevalence studies: modification of an existing tool and evidence of interrater agreement. J Clin Epidemiol. 2012;65(9):934-9. https://doi.org/10.1 016/j.jclinepi.2011.11.014

21. Ackerman IN, Buchbinder R, Osborne RH. Factors limiting participation in arthritis self-management programmes: an exploration of barriers and 
patient preferences within a randomized controlled trial. Rheumatology. 2013:52(3):472-9. https://doi.org/10.1093/rheumatology/kes295.

22. Al-Taiar A, Al-Sabah R, Elsalawy E, Shehab D, Al-Mahmoud S. Attitudes to knee osteoarthritis and total knee replacement in Arab women: a qualitative study. BMC Res Notes. 2013;6(1):406. https://doi.org/10.1186/1 756-0500-6-406

23. Baumann M, Euller-Ziegler L, Guillemin F. Evaluation of the expectations osteoarthritis patients have concerning healthcare, and their implications for practitioners. Clin Exp Rheumatol. 2007;25(3):404-9.

24. Bukhave EB, Huniche L. Activity problems in everyday life - patients' perspectives of hand osteoarthritis: 'Try imagining what it would be like having no hands. Disabil Rehabil. 2014;36(19):1636-43. https://doi.org/10.31 09/09638288.2013.863390.

25. Chan KKW, Chan LWY. A qualitative study on patients with knee osteoarthritis to evaluate the influence of different pain patterns on patients' quality of life and to find out patients' interpretation and coping strategies for the disease. Rheumatol Rep. 2011;3:9-15.

26. Ilori T, Ladipo MM, Ogunbode AM, Obimakinde AM. Knee osteoarthritis and perceived social support amongst patients in a family medicine clinic. S Afr Fam Pract. 2016;58(6):202-6. https://doi.org/10.1080/20786190.2016.1198086.

27. Hill S, Dziedzic KS, Ong BN. The functional and psychological impact of hand osteoarthritis. Chronic IIIness. 2010;6(2):101-10. https://doi.org/10.11 77/1742395309345614.

28. Kao M-H, Tsai Y-F. Illness experiences in middle-aged adults with early-stage knee osteoarthritis: findings from a qualitative study. J Adv Nurs. 2014;70(7): 1564-72. https://doi.org/10.1111/jan.12313.

29. Kjeken I, Darre S, Slatkowsky-Cristensen B, Hermann M, Nilsen T, Eriksen CS et al. Self-management strategies to support performance of daily activities in hand osteoarthritis. Scand J Occup Ther. 2013;20(1):29-36. https://doi. org/10.3109/11038128.2012.661457.

30. Leung YY, Li JC, Thumboo J. Domains rated as important by patients with hand osteoarthritis. Int J Rheum Dis. 2019;22(11):2045-51. https://doi.org/1 0.1111/1756-185X.13709.

31. Neville C, Fortin PR, Fitzcharles M, Baron M, Abrahamowitz M, Du Berger R, et al. The needs of patients with arthritis: The patient's perspective. Arthritis Care Res. 1999:12:85-95.

32. Tanimura C, Morimoto M, Hiramatsu K, Hagino H. Difficulties in the daily life of patients with osteoarthritis of the knee: scale development and descriptive study. J Clin Nurs. 2011;20(5-6):743-53. https://doi.org/10.1111/ j.1365-2702.2010.03536.x.

33. Alten R, van de Laar M, De Leonardis F, Tietz N, Guerreiro M, van Vollenhoven R. Physical and emotional burden of rheumatoid arthritis: data from ra matters, a web-based survey of patients and healthcare professionals. Rheumatol Ther. 2019;6(4):587-97. https://doi.org/10.1007/s40744-019-00179-2.

34. Been-Dahmen JMJ, Walter MJ, Dwarswaard J, Hazes JMW, van Staa A, Ista E. What support is needed to self-manage a rheumatic disorder: a qualitative study. BMC Musculoskelet Disord. 2017;18:1-9.

35. Bergsten U, Bergman S, Fridlund B, Arvidsson B. "Striving for a good life"-the management of rheumatoid arthritis as experienced by patients. Open Nurs J. 2011;5:95-101. https://doi.org/10.2174/1874434601105010095.

36. Carter K, Walmsley S, Chessman D, Rome K, Turner DE. Perspectives of patients and health professionals on the experience of living with psoriatic arthritis-related foot problems: a qualitative investigation. Clin Rheumatol. 2019;38(6):1605-13. https://doi.org/10.1007/s10067-018-04411-2.

37. Cunha-Miranda L, Costa L, Ribeiro JS. Near study: needs and expectations in rheumatoid arthritis - do we know our patients needs? Acta Reumatol. 2010;35:314-23.

38. Giacomelli R, Gorla R, Trotta F, Tirri R, Grassi W, Bazzichi L, et al. Quality of life and unmet needs in patients with inflammatory arthropathies: results from the multicentre, observational rapsodia study. Rheumatology. 2015; 54(5):792-7. https://doi.org/10.1093/rheumatology/keu398.

39. Hamnes B, Hauge MI, Kjeken I, Hagen KB. 'I have come here to learn how to cope with my illness, not to be cured': A qualitative study of patient expectations prior to a one-week self-management programme. Musculoskelet Care. 2011;9(4):200-10. https://doi.org/10.1002/msc.212.

40. Henchoz Y, Zufferey P, So A. Stages of change, barriers, benefits, and preferences for exercise in ra patients: a cross-sectional study. Scand J Rheumatol. 2013;42(2):136-45. https://doi.org/10.3109/03009742.2012. 724707.

41. Herrera-Saray P, Pelaez-Ballestas I, Ramos-Lira L, Sanchez-Monroy D, BurgosVargas R. Usage problems and social barriers faced by persons with a wheelchair and other aids. Qualitative study from the ergonomics perspective in persons disabled by rheumatoid arthritis and other conditions. Reumatology. 2013;9(1):24-30. https://doi.org/10.1016/j.reuma.2 012.05.010.

42. Kostova Z, Caiata-Zufferey M, Schulz PJ. The impact of social support on the acceptance process among ra patients: a qualitative study. Psychol Health. 2014;29(11):1283-302. https://doi.org/10.1080/08870446.2014.925895.

43. Kristiansen TM, Primdahl J, Antoft R, Hørslev-Petersen K. Everyday life with rheumatoid arthritis and implications for patient education and clinical practice: a focus group study. Musculoskelet Care. 2012;10(1):29-38. https:// doi.org/10.1002/msc.224.

44. Laidmae VI, Leppik L, Tulva T, Haal ML. Disease-related social and family life: people coping with rheumatoid arthritis in Estonia. Crit Public Health. 2009; 19(1):87-105. https://doi.org/10.1080/09581590802375871.

45. Lempp H, Scott D, Kingsley G. The personal impact of theumatoid arthritis on patients' identity: a qualitative study. Chronic Illness. 2006;2(2):109-20. https://doi.org/10.1177/17423953060020020601.

46. Sato M, Yamazaki Y, Sakita M, Bryce TJ. Benefit-finding among people with rheumatoid arthritis in Japan. Nurs Health Sci. 2008;10(1):51-8. https://doi. org/10.1111/j.1442-2018.2007.00372.x.

47. Strand V, Wright GC, Bergman MJ, Tambiah J, Taylor PC. Patient expectations and perceptions of goal-setting strategies for disease management in rheumatoid arthritis. J Rheumatol. 2015;42(11):2046-54 https://doi.org/10.3899/jrheum.140976.

48. Sverker A, Ostlund G, Thyberg M, Thyberg I, Valtersson E, Bjork M. Dilemmas of participation in everyday life in early rheumatoid arthritis: a qualitative interview study (the swedish tira project). Disabil Rehabil. 2015:37(14):12519. https://doi.org/10.3109/09638288.2014.961658.

49. Thomas R, Hewlett S, Swales C, Cramp F. Keeping physically active with rheumatoid arthritis: semi-structured interviews to explore patient perspectives, experiences and strategies. Physiotherapy. 2019;105(3):378-84. https://doi.org/10.1016/j.physio.2018.09.001.

50. Van der meer M, Hoving JL, Vermeulen MIM, Herenius MMJ, Tak PP, Sluiter $\mathrm{JK}$, et al. Experiences and needs for work participation in employees with rheumatoid arthritis treated with anti-tumour necrosis factor therapy. Disabil Rehabil. 2011:33:2587-95.

51. Wollenhaupt J, Ehlebracht-Koenig I, Groenewegen A, Fricke D. Prioritizing the patient: optimizing therapy in rheumatoid arthritis. Results of a patient questionnaire in northern Germany. Open Access Rheumatol. 2013;5:51-67. https://doi.org/10.2147/OARRR.S38032.

52. Bank TW. World bank country and lending groups. 2019 [updated 2019; cited]; Available from: https://datahelpdesk.worldbank.org/knowledgebase/a rticles/906519-world-bank-country-and-lending-groups.

53. Lempp H, Hofmann D, Hatch SL, Scott DL. Patients' views about treatment with combination therapy for rheumatoid arthritis: a comparative qualitative study. BMC Musculoskelet Disord. 2012;13(1):200. https://doi.org/10.1186/14 71-2474-13-200.

54. Fitzpatrick R, Newman S, Lamb R, Shipley M. Social relationships and psychological well-being in rheumatoid arthritis. Soc Sci Med. 1988;27(4): 399-403. https://doi.org/10.1016/0277-9536(88)90275-4.

55. Lefevre-Colau MM, Buchbinder R, Regnaux JP, Roren A, Poiraudeau S, Boutron I. Self-management education programmes for rheumatoid arthritis. Cochrane Database Syst Rev. 2014;2014:CD011338.

56. Gignac MA, Cao X, McAlpine J. Availability, need for, and use of work accommodations and benefits: are they related to employment outcomes in people with arthritis? Arthritis Care Res. 2015;67(6):855-64. https://doi org/10.1002/acr.22508.

57. Barlow J, Wright C, Kroll T. Overcoming perceived barriers to employment among people with arthritis. J Health Psychol. 2001;6(2):205-16. https://doi. org/10.1177/135910530100600201.

58. Wilcox S, Der Ananian C, Abbott J, Vrazel J, Ramsey C, Sharpe PA, et al. Perceived exercise barriers, enablers, and benefits among exercising and nonexercising adults with arthritis: results from a qualitative study. Arthritis Rheum. 2006;55(4):616-27. https://doi.org/10.1002/art.22098.

59. Dobson F, Bennell KL, French SD, Nicolson PJ, Klaasman RN, Holden MA, et al. Barriers and facilitators to exercise participation in people with hip and/or knee osteoarthritis: synthesis of the literature using behavior change theory. Am J Phys Med Rehabil. 2016;95(5):372-89. https://doi.org/10.1097/ PHM.0000000000000448.

60. Rausch Osthoff AK, Niedermann K, Braun J, Adams J, Brodin N, Dagfinrud H, et al. 2018 eular recommendations for physical activity in people with 
inflammatory arthritis and osteoarthritis. Ann Rheum Dis. 2018;77(9):125160. https://doi.org/10.1136/annrheumdis-2018-213585.

61. Commission P. Caring for older australians, report no. 53, final inquiry report. Canberra; 2011. Contract No.: Document Number

62. Ackerman IN, Jordan JE, Van Doornum S, Ricardo M, Briggs AM. Understanding the information needs of women with rheumatoid arthritis concerning pregnancy, post-natal care and early parenting: a mixedmethods study. BMC Musculoskelet Disord. 2015;16(1):194. https:/doi.org/1 0.1186/s12891-015-0657-4.

63. Britten N, Ekman I, Naldemirci O, Javinger M, Hedman H, Wolf A. Learning from Gothenburg model of person centred healthcare. BMJ. 2020;370: m2738.

\section{Publisher's Note}

Springer Nature remains neutral with regard to jurisdictional claims in published maps and institutional affiliations.

Ready to submit your research? Choose BMC and benefit from:

- fast, convenient online submission

- thorough peer review by experienced researchers in your field

- rapid publication on acceptance

- support for research data, including large and complex data types

- gold Open Access which fosters wider collaboration and increased citations

- maximum visibility for your research: over $100 \mathrm{M}$ website views per year

At $B M C$, research is always in progress.

Learn more biomedcentral.com/submissions 